\title{
Homogenization of the free boundary velocity
}

\author{
Inwon C. Kim
}

February 17, 2006

\begin{abstract}
In this paper we investigate some free boundary problems with space-dependent free boundary velocities. Based on maximum principletype arguments, we show the uniform convergence of the solutions in the homogenization limit. The main step is to show the uniqueness of the limiting free boundary velocity, which turns out to be a continuous function of the normal direction of the free boundary.
\end{abstract}

\section{Introduction}

Consider a compact set $K \subset \mathbb{R}^{n}$ with smooth boundary $\partial K$. Suppose that a bounded domain $\Omega$ contains $K$ and let $\Omega_{0}=\Omega-K$ and $\Gamma_{0}=\partial \Omega$. Note that $\partial \Omega_{0}=\Gamma_{0} \cup \partial K$. Let $u_{0}$ be the harmonic function in $\Omega_{0}$ with $u_{0}=f>0$ on $K$ and zero on $\Gamma_{0}$. (see Figure 1.)

Let us define $e_{i} \in \mathbb{R}^{n}, i=1, \ldots, n$ such that

$$
e_{1}=(1,0, . .0), e_{2}=(0,1,0, . ., 0), \ldots, \text { and } e_{n}=(0, . ., 0,1) .
$$

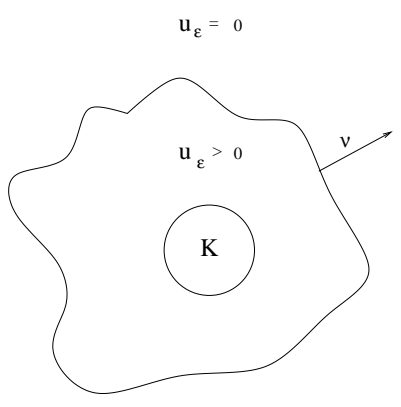

Figure 1. 
Consider a continuous function

$$
g: \mathbb{R}^{n} \rightarrow[1,2], \quad g\left(x+e_{i}\right)=g(x) \text { for } i=1, \ldots, n .
$$

In this paper we consider the behavior, as $\epsilon \rightarrow 0$, of the nonnegative (viscosity) solutions $u^{\epsilon} \geq 0$ of the following problem

$$
(P)_{\epsilon} \quad \begin{cases}-\Delta u^{\epsilon}=0 & \text { in }\left\{u^{\epsilon}>0\right\}, \\ u_{t}^{\epsilon}-g\left(\frac{x}{\epsilon}\right)\left|D u^{\epsilon}\right|^{2}=0 & \text { on } \partial\left\{u^{\epsilon}>0\right\}\end{cases}
$$

in $Q=\left(\mathbb{R}^{n}-K\right) \times(0, \infty)$ with initial data $u_{0}$ and fixed boundary data $f=1$ on $\partial K \times[0, \infty)$. Here $D u$ denotes the spatial derivative of $u$.

We refer to $\Gamma_{t}\left(u^{\epsilon}\right):=\partial\left\{u^{\epsilon}(\cdot, t)>0\right\}-\partial K$ as the free boundary of $u^{\epsilon}$ at time $t$ and to $\Omega_{t}\left(u^{\epsilon}\right):=\left\{u^{\epsilon}(\cdot, t)>0\right\}$ as the positive phase of $u^{\epsilon}$. Note that if $u^{\epsilon}$ is smooth up to the free boundary, then the free boundary moves with normal velocity $V=u_{t}^{\epsilon} /\left|D u^{\epsilon}\right|$, and hence the second equation in $(P)_{\epsilon}$ implies that

$$
V=g\left(\frac{x}{\epsilon}\right)|D u|=g\left(\frac{x}{\epsilon}\right)\left(D u^{\epsilon} \cdot(-\nu)\right),
$$

where $\nu=\nu_{(x, t)}$ denotes the outward normal vector at the free boundary point $x \in \Gamma_{t}(u)$ with respect to $\Omega_{t}(u)$.

Free boundary problems with space-dependent velocity law as in $(P)_{\epsilon}$ describe various motions in heterogeneous media, including heat transfer $[\mathrm{P}],[\mathrm{R}]$, contact line dynamics of fluids $[\mathrm{G}]$, and shoreline movements in oceanography [VSKP].

Our main result is that there exists a unique motion law of the free boundary in the homogenization limit as $\epsilon \rightarrow 0$. More precisely we will show that there exists a continuous function $r(x)$ on $\left\{x \in \mathbb{R}^{n}:|x|=1\right\}$ such that the family of solutions $\left\{u^{\epsilon}\right\}$ of $(P)_{\epsilon}$ uniformly converges to $u$, where $u$ satisfies

$$
\begin{cases}-\Delta u=0 & \text { in }\{u>0\} \\ u_{t}-r(\nu)\left|D u^{\epsilon}\right|^{2}=0 & \text { on } \partial\{u>0\}\end{cases}
$$

in $Q$ (Theorem 4.2).

We mention that the method presented in this paper applies to the general case of continuous function $g$ with range $0<a \leq g \leq b<\infty$, and strictly positive, smooth fixed boundary data $f=f(x, t)$ on $\partial K$. However the fact that the positive phase strictly expands $(g>0)$ is essential in our proof. 
There are vast amount of literature on the subject of homogenization. For detailed survey on different approaches we refer to Caffarelli, Souganidis and Wang [CSW]. Papanicolaou and Varadhan [PV] and Kozlov [Ko] were the first to consider the problem of homogenizing linear, uniformly elliptic and parabolic operators. The first nonlinear result in the variational setting was obtained by Dal Maso and Modica [DM]. For fully nonlinear, uniformly elliptic and parabolic operators, Evans [E] and Caffarelli $[\mathrm{C}]$ derived convergence results using viscosity solutions, which was first introduced by Crandall and Lions for studying Hamilton-Jacobi equations (see for example [CIL]).

For free boundary problems, very few homogenization results are proven due to the lower-dimensional nature of the interface. For example, the periodicity of $g$ in $(P)_{\epsilon}$ does not guarantee the interface $\Gamma_{t}(u)$ to be periodic in space. Moreover the restriction of $g$ on the interface and the propagation of the interface affects each other, which makes the analysis challenging even if we assume that the interface is smooth.

Recently the uniform convergence of pulsating traveling fronts of the flame-propagation type free boundary problem

$(F P)_{\epsilon}$

$$
\begin{cases}u_{t}^{\epsilon}-\Delta u^{\epsilon}=0 & \text { in }\left\{u^{\epsilon}>0\right\}, \\ \left|D u^{\epsilon}\right|^{2}=f\left(\frac{x}{\epsilon}\right) & \text { on } \partial\left\{u^{\epsilon}>0\right\}\end{cases}
$$

has been studied by Caffarelli, Lee and Mellet [CLM1], [CLM2]. Here to avoid analysis on the interface, $(F P)_{\epsilon}$ is approximated by the phase-field model

$$
(P)_{\delta, \epsilon} \quad u_{t}^{\epsilon, \delta}-\Delta u^{\epsilon, \delta}=f\left(\frac{x}{\epsilon}\right) \frac{1}{\delta} \beta\left(\frac{u^{\epsilon, \delta}}{\delta}\right)
$$

where $\beta$ is a smooth function whose support is $[0,1]$ with $\int_{0}^{1} \beta=1$ and $0<\delta<<\epsilon$. Existence, uniqueness and regularity properties of the pulsating traveling fronts of $(P)_{\delta, \epsilon}$, shown by Berestycki and Hamel [BH], is essential in the investigation.

The novelty of our approach is that we directly deal with the presence of the free boundary in $(P)_{\epsilon}$ using viscosity solutions. We apply maximum principle-type arguments and stability property of viscosity solutions, without any regularity estimate or approximation on the solutions of $(P)_{\epsilon}$, to prove the uniform convergence results and properties of solutions in the homogenization limit. To define the limiting free boundary velocity we apply the ideas in $[\mathrm{CSW}]$, which studies homogenization limits of fully nonlinear 
equations in ergodic random media. The main idea in the analysis of [CSW] is that, to describe the limiting problem, it is enough to decide whether a given 'test function' is either a subsolution or a supersolutions of the problem. The test functions were quadratic polynomials in [CSW] since the equation under investigation was of second-order, but for our problem, whose motion law is of first order, the test functions are the linear blowup profiles $P_{q, r}$, which is planar front propagations with given speed $r$ and normal direction $q \in \mathbb{R}^{n}$ of the propagation (see section 2 ).

As mentioned before, the presence of the free boundary is the central difficulty in applying the idea introduced in [CSW].

Note that the linear blow-up of the solutions of $(F P)_{\epsilon}$ leads to the stationary problem

$$
-\Delta u^{\epsilon}=0 \text { in }\left\{u^{\epsilon}>0\right\} ; \quad|D u|^{2}=f\left(\frac{x}{\epsilon}\right) \text { on } \partial\left\{u^{\epsilon}>0\right\},
$$

which does not have a unique solution (see the numerical experiment in [CLM2] where, with linear blow-up, the free boundary of $(F P)_{\epsilon}$ jumps from one state to another). In our problem the linear blow-up preserves the speed of free boundary propagation, which suggests more stability. In fact the continuity of the limiting free boundary condition with respect to the normal direction does not hold in the limit of $(F P)_{\epsilon}$ (see Appendix 2 of [CLM2].)

Below we give the outline of the paper.

In section 1 we introduce the notion of viscosity solutions for $(P)_{\epsilon}$, extended from $[\mathrm{K} 1]$, and their properties.

In section 2, we define and study properties of maximal sub- and minimal supersolutions of $(P)_{\epsilon}$ for given obstacle $P_{q, r}$. An obstacle $P_{q, r}$ is a 'subsolution' for the limit problem if the maximal subsolution below $P_{q, r}$ converges to the obstacle as $\epsilon \rightarrow 0$, and similarly an obstacle $P_{q, r}$ is a 'supersolution' for the limit problem if the minimal supersolution above $P_{q, r}$ converges to the obstacle in the limit. The goal is to find a unique obstacle $P_{q, r}$ which serves for both sub- and supersolution of the limit problem, for each given normal direction $q$.

In section 3 , we prove that this is indeed possible. In other words, we show that, for given normal unit vector $q \in \mathbb{R}^{n}$, there is a unique speed $r=r(q)$ such that both the maximal sub- and minimal supersolution of $(P)_{\epsilon}$ with obstacle $P_{q, r}$ converge to $P_{q, r}$ as $\epsilon \rightarrow 0$. This $r(q)$ will be our candidate for the function $r$ given in the free boundary motion law in $(P)$. We also prove that $r(q)$ is continuous with respect to the normal direction $q$ of the obstacle. The flatness of the free boundary of the maximal sub- and 
minimal supersolution (Lemma 2.9), with a 'good' obstacle, is central to the analysis. To prove the uniqueness of $r(q)$, for rational normal directions we use the periodicity of interface of the global solutions (see the proof of Lemma 3.2 and Proposition 3.4), and for non-rational normal vectors we use the fact that rotations by irrational angels generate a dense image on the circle (see the proof of Lemma 3.8).

In section 4 , it is shown that $r(q)$ obtained in section 3 indeed yields the limiting free boundary motion law $V=r(\nu)|D u|$ in $(P)$. The uniform convergence of $\left\{u^{\epsilon}\right\}$ then follows from the comparison principle (Theorem 1.7) for $(P)$.

Section 5 is on the homogenization of Stefan-type problem $(P 2)_{\epsilon}$, which replaces the Laplace operator in $(P)_{\epsilon}$ with the heat operator. We observe that the linear blow-up of the heat operator generates the Laplace operator, which suggests that the limiting free boundary motion law for $(P 2)_{\epsilon}$ may be the same as in the case of $(P)_{\epsilon}$. This is indeed what we prove in this section.

Acknowledgements: The author thanks Takis Souganidis for suggesting investigation of free boundary motions with space-dependent velocity, which motivated this paper. The author is also grateful to Luis Caffarelli for his inspiring lectures on nonlinear homogenization at University of Texas-Austin when the author was a student.

\section{$1 \quad$ Viscosity solutions}

Let $g$ be a continuous function

$$
g(x, y): \mathbb{R}^{n} \times\left\{y \in \mathbb{R}^{n}:|y|=1\right\} \rightarrow[1,2]
$$

with the property

$$
g\left(x+e_{i}, y\right)=g(x, y) \quad \text { for } i=1, \ldots, n,
$$

where $e_{i} \in \mathbb{R}^{n}, i=1, \ldots, n$ is given in (0.1).

We consider the free boundary problem

$(\tilde{P})_{\epsilon} \quad \begin{cases}-\Delta u=0 & \text { in }\{u>0\}, \\ u_{t}-g\left(\frac{x}{\epsilon}, \nu\right)\left|D u^{\epsilon}\right|^{2}=0 & \text { on } \partial\{u>0\}\end{cases}$

where $\nu=\nu_{x, t}$ is the outward spatial normal vector at $(x, t) \in \partial\{u>0\}$ with respect to $\{u>0\}$, as given in the introduction. 
Note that $g(x, y) \equiv g(x)$ in $(P)_{\epsilon}$ defined in the introduction and $g(x, y)=$ $r(y)$ in the limit problem $(P)$ defined in section 4 . In this section we prove existence and uniqueness results for the generalized problem $(\tilde{P})_{\epsilon}$ to apply the results to both $(P)_{\epsilon}$ and $(P)$.

We extend the notion of viscosity solutions of Hele-Shaw problem $(g \equiv 1$ in $\left.(\tilde{P})_{\epsilon}\right)$ introduced in [K1]. Roughly speaking viscosity sub and supersolutions are defined by comparison with local (smooth) super and subsolutions. In particular classical solutions of $(\tilde{P})_{\epsilon}$ are also viscosity sub and supersolutions of $(\tilde{P})_{\epsilon}$.

Consider a domain $D \subset \mathbb{R}^{n}$ and an interval $I \subset \mathbb{R}$. For a nonnegative real valued function $u(x, t)$ defined for $(x, t) \in D \times I$, define

$$
\begin{aligned}
& \Omega(u)=\{(x, t) \in D \times I: u(x, t)>0\}, \quad \Omega_{t}(u)=\{x \in D: u(x, t)>0\} ; \\
& \Gamma(u)=\partial \Omega(u)-\partial(D \times I), \quad \Gamma_{t}(u)=\partial \Omega_{t}(u)-\partial D .
\end{aligned}
$$

Let $Q$ and $K$ be as given in the introduction and let $\Sigma$ be a cylindrical domain $D \times(a, b) \subset \mathbb{R}^{n} \times \mathbb{R}$, where $D$ is an open subset of $\mathbb{R}^{n}$.

Definition 1.1. A nonnegative upper semicontinuous function $u$ defined in $\Sigma$ is a viscosity subsolution of $(\tilde{P})_{\epsilon}$ if

(a) for each $a<T<b$ the set $\overline{\Omega(u)} \cap\{t \leq T\} \cap \Sigma$ is bounded; and

(b) for every $\phi \in C^{2,1}(\Sigma)$ such that $u-\phi$ has a local maximum in $\overline{\Omega(u)} \cap$ $\left\{t \leq t_{0}\right\} \cap \Sigma$ at $\left(x_{0}, t_{0}\right)$ then

(i) $-\Delta \phi\left(x_{0}, t_{0}\right) \leq 0 \quad$ if $u\left(x_{0}, t_{0}\right)>0$.

(ii) $\quad$ if $\left(x_{0}, t_{0}\right) \in \Gamma(u),|\nabla \phi|\left(x_{0}, t_{0}\right) \neq 0$ and $-\Delta \varphi\left(x_{0}, t_{0}\right)>0$

then

$$
\left(\phi_{t}-g\left(\frac{x_{0}}{\epsilon}, \nu\right)|D \phi|^{2}\right)\left(x_{0}, t_{0}\right) \leq 0,
$$

where $\nu=-\frac{D \phi}{|D \phi|}\left(x_{0}, t_{0}\right)$.

Note that because $u$ is only upper semicontinuous there may be points of $\Gamma(u)$ at which $u$ is positive. 
Definition 1.2. A nonnegative lower semicontinuous function $v$ defined in $\Sigma$ is a viscosity supersolution of $(\tilde{P})_{\epsilon}$ if for every $\phi \in C^{2,1}(\Sigma)$ such that $v-\phi$ has a local minimum in $\Sigma \cap\left\{t \leq t_{0}\right\}$ at $\left(x_{0}, t_{0}\right)$, then

$$
\begin{gathered}
\text { (i) } \quad-\Delta \phi\left(x_{0}, t_{0}\right) \geq 0 \quad \text { if } v\left(x_{0}, t_{0}\right)>0, \\
\text { (ii) } \quad \text { if }\left(x_{0}, t_{0}\right) \in \Gamma(v),|\nabla \phi|\left(x_{0}, t_{0}\right) \neq 0 \text { and } \\
-\Delta \varphi\left(x_{0}, t_{0}\right)<0,
\end{gathered}
$$

then

$$
\left(\phi_{t}-g\left(\frac{x_{0}}{\epsilon}, \nu\right)|\nabla \phi|^{2}\right)\left(x_{0}, t_{0}\right) \geq 0 .
$$

where $\nu=-\frac{D \phi}{|D \phi|}\left(x_{0}, t_{0}\right)$.

Definition 1.3. $u$ is a viscosity subsolution of $(\tilde{P})_{\epsilon}$ with initial data $u_{0}$ and fixed boundary data $f>0$ if

(a) $u$ is a viscosity subsolution in $Q$,

(b) $u$ is upper semicontinuous in $\bar{Q}, u=u_{0}$ at $t=0$ and $u \leq f$ on $\partial K$.

(c) $\overline{\Omega(u)} \cap\{t=0\}=\overline{\Omega\left(u_{0}\right)}$.

Definition 1.4. $u$ is a viscosity supersolution of $(\tilde{P})_{\epsilon}$ with initial data $u_{0}$ and fixed boundary data $f$ if $u$ is a viscosity supersolution in $Q$, lower semicontinuous in $\bar{Q}$ with $u=u_{0}$ at $t=0$ and $u \geq f$ on $\partial K$.

For a nonnegative real valued function $u(x, t)$ in a cylindrical domain $D \times(a, b)$ we define

$$
u^{*}(x, t):=\limsup _{(\xi, s) \in D \times(a, b) \rightarrow(x, t)} u(\xi, s) .
$$

and

$$
u_{*}(x, t):=\liminf _{(\xi, s) \in D \times(a, b) \rightarrow(x, t)} u(\xi, s) .
$$

Definition 1.5. $u$ is a viscosity solution of $(\tilde{P})_{\epsilon}$ (with boundary data $u_{0}$ and $f$ ) if $u$ is a viscosity supersolution and $u^{*}$ is a viscosity subsolution of $(\tilde{P})_{\epsilon}\left(\right.$ with boundary data $u_{0}$ and $f$.)

Definition 1.6. We say that a pair of functions $u_{0}, v_{0}: \bar{D} \rightarrow[0, \infty)$ are (strictly) separated (denoted by $u_{0} \prec v_{0}$ ) in $D \subset \mathbb{R}^{n}$ if 
(i) the support of $u_{0}, \operatorname{supp}\left(u_{0}\right)=\overline{\left\{u_{0}>0\right\}}$ restricted in $\bar{D}$ is compact and

(ii) in $\operatorname{supp}\left(u_{0}\right) \cap \bar{D}$ the functions are strictly ordered:

$$
u_{0}(x)<v_{0}(x) .
$$

Theorem 1.7. (Comparison principle) Let $u, v$ be respectively viscosity suband supersolutions of $(\tilde{P})_{\epsilon}$ in $D \times(0, T) \subset Q$ with $u(\cdot, 0) \prec v(\cdot, t)$ in $D$. If $u \prec v$ on $\partial D$ for $0 \leq t<T$, then $u(\cdot, t) \prec v(\cdot, t)$ in $D$ for $t \in[0, T)$.

The proof is parallel to the proof of Theorem 2.2 in [K1]. We only sketch the outline of the proof below.

\section{Sketch of the proof}

1. For $r, \delta>0$ and $0<h<<r$, define the sup-convolution of $u$

$$
Z(x, t):=(1+\delta) \sup _{|(y, s)-(x, t)|<r} u(y, s)
$$

and the inf-convolution of $v$

$$
W(x, t):=(1-\delta) \inf _{|(y, s)-(x, t)|<r-h t} v(y, s)
$$

in $\tilde{D} \times[r, r / h], \tilde{D}:=\{x: x \in D, d(x, \partial D) \geq r\}$.

By upper semi-continuity of $u-v, Z(\cdot, r) \prec W(\cdot, r)$ for sufficiently small $r, \delta>0$. Moreover a parallel argument as in the proof of Lemma 1.3 in [K1] yields that if $r<<\delta \epsilon, Z$ and $W$ are respectively sub- and supersolutions of $(\tilde{P})_{\epsilon}$.

2. By our hypothesis and the upper semi-continuity of $u-v, Z \leq W$ on $\partial \tilde{D}$ and $Z<W$ on $\partial \tilde{D} \cap \bar{\Omega}(Z)$ for sufficiently small $\delta$ and $r$. If our theorem is not true for $u$ and $v$, then $Z$ crosses $W$ from below at $P_{0}:=$ $\left(x_{0}, t_{0}\right) \in \tilde{D} \times[r, T]$. Due to the maximum principle of harmonic functions, $P_{0} \in \Gamma(Z) \cap \Gamma(W)$. Note that by definition $\Omega(Z)$ and $\Omega(W)$ has respectively an interior ball $B_{1}$ and exterior ball $B_{2}$ at $P_{0}$ of radius $r$ in space-time (see Figure 2.)

3. Let us call $H$ the tangent hyperplane to the interior ball of $Z$ at $P_{0}$. Since $Z \leq W$ for $t \leq t_{0}$ and $P_{0} \in \Gamma(Z) \cap \Gamma(W)$, it follows that

$$
B_{1} \cap\left\{t \leq t_{0}\right\} \subset \Omega(Z) \cap \Omega(W) ; \quad B_{2} \cap\left\{t \leq t_{0}\right\} \subset\{Z=0\} \cap\{W=0\}
$$

with $\bar{B}_{1} \cap \bar{B}_{2} \cap\left\{t \leq t_{0}\right\}=\left\{P_{0}\right\}$.

Moreover, since $Z$ and $W$ respectively satisfies the free boundary motion law

$$
\frac{Z_{t}}{|D Z|}(x, t) \leq g\left(\frac{x}{\epsilon}, \nu\right)|D Z|(x, t) \leq 2|D Z|(x, t) \text { on } \Gamma(Z)
$$




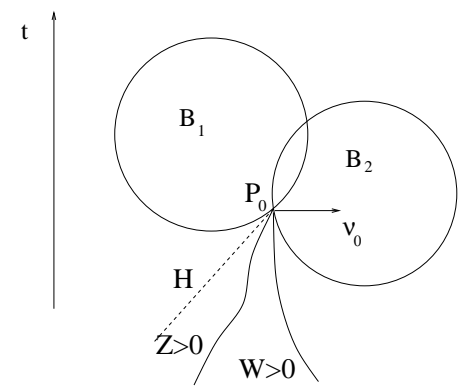

Figure 2.

and

$$
\frac{W_{t}}{|D W|}(x, t) \geq g(x / \epsilon, \nu)|D W|(x, t)+h \geq|D W|(x, t)+h \text { on } \Gamma(W),
$$

the arguments of Lemma 2.5 in [K1] applies for $Z$ to yield that $H$ is neither vertical nor horizontal. In particular $B_{1} \cap\left\{t=t_{0}\right\}$ and $B_{2} \cap\left\{t=t_{0}\right\}$ share the same normal vector $\nu_{0}$, outward with respect to $B_{1}$, at $P_{0}$.

Formally speaking, it follows that

$$
\frac{Z_{t}}{|D Z|}\left(P_{0}\right) \leq g\left(x_{0} / \epsilon, \nu_{0}\right)|D Z|\left(P_{0}\right) \leq g\left(x_{0} / \epsilon, \nu_{0}\right)|D W|\left(P_{0}\right) \leq \frac{W_{t}}{|D W|}\left(p_{0}\right)-h,
$$

where the second inequality follows since $Z\left(\cdot, t_{0}\right) \leq W\left(\cdot, t_{0}\right)$ in a neighborhood of $x_{0}$. Above inequality says that the free boundary speed of $Z$ is strictly less than that of $W$ at $P_{0}$, contradicting the fact that $\Gamma(Z)$ touches $\Gamma(W)$ from below at $P_{0}$.

(For rigorous argument one can construct barrier functions based on the exterior and interior ball properties of $Z$ and $W$ at $P_{0}$. For details see the proof of Theorem 2.2 in [K1].)

For $x \in \mathbb{R}^{n}$, we denote $B_{r}(x):=\left\{y \in \mathbb{R}^{n}:|y-x|<r\right\}$. For simplicity, in this paper we will consider solutions with fixed boundary data $f=1$ and the fixed boundary $\partial K=\partial B_{1}(0)$. Let $u_{0}, \Omega_{0}$ and $\Gamma_{0}$ as in the introduction.

Theorem 1.8. (a) If Int $\left(\bar{\Omega}_{0}\right)=\Omega_{0}$ and if $\Omega\left(u_{0}\right)$ immediately expands, in other words if $\Gamma_{0} \subset$ int $\Omega_{t}(u)$ for $t>0$ and for any viscosity solution $u$ of $(\tilde{P})_{\epsilon}$ with initial data $u_{0}$, then there is a unique viscosity solution $u$ of $(\tilde{P})_{\epsilon}$ with initial data $u_{0}$. 
(b) $u$ is increasing in $t, u(\cdot, t)$ is harmonic in $\Omega_{t}(u), u^{*}(\cdot, t)$ is harmonic in $\Omega_{t}\left(u^{*}\right)$, and $\Gamma\left(u^{*}\right)=\Gamma(u)$.

Proof. 1. Since $\Omega\left(u_{0}\right)$ immediately expands, for any $\delta>0$ and for any two viscosity solutions $u_{1}$ and $u_{2}$ of $(\tilde{P})_{\epsilon}$ with initial data $u_{0}$,

$$
u_{1}(x, 0) \prec(1+\delta) u_{2}(x, \delta) \text { at } t=0 .
$$

By Theorem 1.7,

$$
u_{1}^{*}(x, t) \prec(1+\delta) u_{2}(x,(1+\delta) t+\delta) \text { for } t>0 .
$$

We now send $\delta \rightarrow 0$ to obtain $u_{1}^{*} \leq u_{2}$, and similarly $u_{2}^{*} \leq u_{1}$, and thus $u_{1}=u_{2}$, yielding uniqueness. For existence, let us consider $\Psi$ : the viscosity solution of $(\tilde{P})_{\epsilon}$ with $g \equiv 2$, with initial data $u_{0}$ and fixed boundary data 1 on $\partial K$ - such solution exists and is unique due to [K1]. Note that $\Psi$ is a supersolution of $(\tilde{P})_{\epsilon}$, for any $g(x, \nu)$ with $g \in[1,2]$. If we let

$U:=\sup \left\{z: z\right.$ is a subsolution of $(\tilde{P})_{\epsilon}, z=1$ on $\partial K, \Gamma_{0}(z)=\Gamma_{0}$, and $\left.z \leq \Psi\right\}$,

Then arguing as in Theorem 4.7 in [K1] will yield that $U_{*}$ is in fact a viscosity solution of $(\tilde{P})_{\epsilon}$ with boundary data $\Gamma_{0}$ and 1 on $\partial K$. We mention that the continuity of $g$ is necessary to prove that $U_{*}$ is a supersolution.

2. For (b) parallel arguments as in the proof of Lemma 1.9 of [K2] applies. In particular

$$
u(\cdot, t)=\inf \left\{\alpha(x):-\Delta \alpha \geq 0 \text { in } \Omega_{t}(u)-K, \alpha=1 \text { on } \partial K, \alpha \geq 0 \text { on } \Gamma_{t}(u) .\right\}
$$

and

$u^{*}(\cdot, t)=\sup \left\{\beta(x):-\Delta \beta \leq 0\right.$ in $\Omega_{t}\left(u^{*}\right)-K, \beta=1$ in $\partial K, \beta \geq 0$ in $\left.\Gamma_{t}\left(u^{*}\right).\right\}$

For later use we show that the free boundary of a viscosity solution $u$ of $(\tilde{P})_{\epsilon}$ in $Q$ with boundary data $u_{0}$ and $f=1$ on $\partial K$ does not jump in time.

Lemma 1.9. $\Gamma(u)$ does not jump in time, in the sense that for any point $x_{0} \in \Gamma_{t_{0}}\left(u^{*}\right)\left(x_{0} \in \Gamma_{t_{0}}(u)\right)$ there exists a sequence of points $\left(x_{n}, t_{n}\right) \in \Gamma\left(u^{*}\right)$ $\left(\left(x_{n}, t_{n}\right) \in \Gamma(u)\right)$ such that $t_{n}<t_{0}\left(t_{n}>t_{0}\right),\left(x_{n}, t_{n}\right) \rightarrow\left(x_{0}, t_{0}\right)$. 
Proof. We only prove the lemma for $u^{*}$. Suppose the lemma is not true. Then for some $x_{0} \in \Gamma_{t_{0}}\left(u^{*}\right)$ there exists $r>0$ such that $B_{2 r}\left(x_{0}\right) \subset\left\{u^{*}(\cdot, t)=\right.$ $0\}$ for $t<t_{0}$. For $\tau>0$ construct a function $\phi$ in $B_{2 r}\left(x_{0}\right) \times\left[t_{0}-\tau, t_{0}\right]$ such that

$$
\begin{cases}-\Delta \phi(\cdot, t)=0 & \text { in } \quad B_{2 r}\left(x_{0}\right)-B_{r(t)}\left(x_{0}\right) ; \\ \phi=\sup _{B_{2 r}\left(x_{0}\right) \times\left[t_{0}-\tau, t\right]} u^{*} \leq 1 & \text { on } \quad \partial B_{2 r}\left(x_{0}\right) \times\left[t-t_{0}, t\right] \\ \phi(\cdot, t)=0 & \text { in } \quad \bar{B}_{r(t)}\left(x_{0}\right),\end{cases}
$$

where $r(t):=\left(1-\tau^{-1}\left(t-t_{0}+\tau\right) / 2\right) r$.

If we choose $\tau>0$ sufficiently small, $\phi$ is a supersolution of $(\tilde{P})_{\epsilon}$ and it follows from Theorem 1.7 that $\Gamma\left(u^{*}\right)$ does not reach $x_{0}$ by time $t=t_{0}$, a contradiction.

\section{Defining the limiting velocity}

In this section we follow ideas from [CSW] to define the limiting free boundary velocity of the solutions of $(P)_{\epsilon}$ as $\epsilon \rightarrow 0$. Roughly speaking, the limiting free boundary velocity is defined by classifying planar propagations into suband supersolutions, depending on how close the sub- and supersolutions of $(P)_{\epsilon}$ placed below or above the obstacle approaches the obstacle in the limit.

For given nonzero vector $q \in \mathbb{R}^{n}$ and $r \in \mathbb{R}^{+}$, we denote

$$
P_{q, r}(x, t):=(r t-q \cdot x)_{+}, \quad l_{q, r}(t)=\left\{x \in \mathbb{R}^{n}: r t=x \cdot q\right\}
$$

Note that the free boundary of $P_{q, r}, \Gamma_{t}\left(P_{q, r}\right):=l_{q, r}(t)$, propagates with normal velocity $r /|q|$ with its outward normal direction $q$, and with $l_{q, r}(0)=\{x: q \cdot x=0\}$.

In $e_{1}-e_{n}$ plane, consider a vector $\mu=e_{n}+\sqrt{3} e_{1}$. Let $l$ to be the line which is parallel to $\mu$ and passes through $3 e_{1}$. Rotate $l$ with respect to $e_{n}$-axis and Let $\mathcal{D}$ to be the region bounded by the rotated image and $\left\{x:-1 \leq x \cdot e_{n} \leq 6.\right\}$ (see Figure 3.) For any nonzero vector $q \in \mathbb{R}^{n}$, let us define $\mathcal{D}(q):=\Psi(\mathcal{D})$, where $\Psi$ is a rotation in $\mathbb{R}^{n}$ which maps $e_{n}$ to $q /|q|$.

Definition 2.1. Let $Q_{1}:=\mathcal{D}(q) \times[0,1]$.

$$
\begin{aligned}
& \bar{u}_{\epsilon ; q, r}:=\left(\sup \left\{u: \text { a subsolution of }(P)_{\epsilon} \text { in } Q_{1} \text {, with } u \leq P_{q, r}\right\}\right)^{*} \\
& \underline{u}_{\epsilon ; q, r}:=\left(\inf \left\{v: \text { a supersolution of }(P)_{\epsilon} \text { in } Q_{1} \text { with } u \geq P_{q, r}\right\}\right)_{*}
\end{aligned}
$$




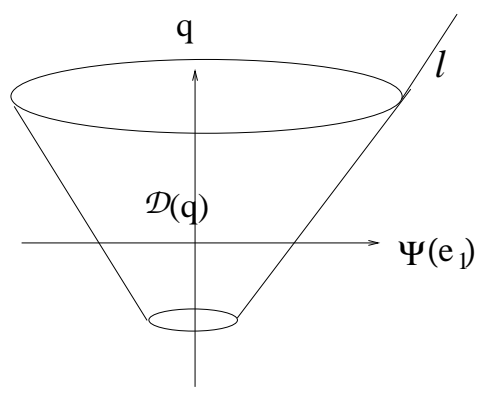

Figure 3.

Remark The reason for defining rather complicated domain $\mathcal{D}(q)$ rather than $B_{1}(0)$ is to guarantee that the free boundary of $\bar{u}_{\epsilon ; q, r}$ and $\underline{u}_{\epsilon ; q, r}$ does not detach from $P_{q, r}$ as it gets away from the lateral boundary of $\mathcal{D}(q)$ too fast. (see Corollary 2.4).

Lemma 2.2. (a) For any $a>0$,

$$
\bar{u}_{\epsilon ; a q, r}(x, t)=a \bar{u}_{\epsilon ; q, a^{-2} r}(x, a t) .
$$

and

$$
\underline{u}_{\epsilon ; a q, r}(x, t)=a \underline{u}_{\epsilon ; q, a^{-2} r}(x, a t)
$$

(b) For $r \geq 2|q|^{2}, P_{q, r}$ is a supersolution of $(P)_{\epsilon}$. For $r \leq|q|^{2}, P_{q, r}$ is a subsolution of $(P)_{\epsilon}$.

Proof. (a) follows from the scaling properties of $(P)_{\epsilon}$. (b) is due to our hypothesis: $1 \leq g \leq 2$.

Due to Lemma 2.2 we are able to restrict the investigation of properties of $\bar{u}_{\epsilon ; q, r}$ and $\underline{u}_{\epsilon ; q, r}$ to the case $|q|=1$ and $r \in[1,2]$.

Next we investigate the behavior of $\bar{u}_{\epsilon ; q, r}$ and $\underline{u}_{\epsilon ; q, r}$ near the lateral boundary of $\mathcal{D}(q) \times[0,1]$. For this we need to construct barriers $\underline{U}_{q, r}$ and $\bar{U}_{q, r}$ to compare respectively with $\underline{u}_{\epsilon ; q, r}$ and $\bar{u}_{\epsilon ; q, r}$.

In $e_{1}-e_{n}$ plane, for each $0 \leq t \leq 1$ consider a line $l(t)$ which is parallel to the vector $e_{1}+\sqrt{3} e_{n}$ and passes through $-e_{1}+t e_{n}$. Now rotate

$$
l(t) \cap\left\{x \cdot e_{1} \leq 0\right\}
$$

with respect to $e_{n}$-axis to obtain a hyper-surface $\underline{L}(t)$ in $\mathbb{R}^{n}$. Let $\mathcal{L}$ be the region whose boundary is $\underline{L}(t)$ and contains $-e_{n}$. For a unit vector $q \in \mathbb{R}^{n}$ let us define $\mathcal{L}(q)=\Phi(\mathcal{L})$ where $\Phi$ is the rotation map in $\mathbb{R}^{n}$ such that $\Phi\left(e_{n}\right)=q($ see Figure 4$)$. 


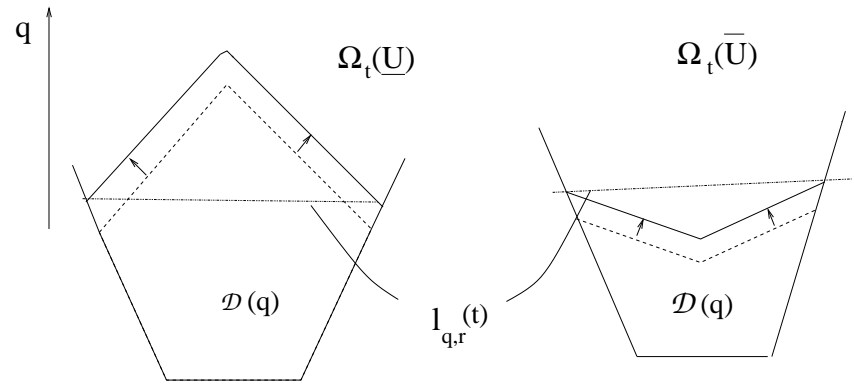

Figure 4.

For $0 \leq t \leq 1$ define $\bar{U}_{q}(\cdot, t)$ to be the harmonic function in the region $\mathcal{L}(q) \cap \mathcal{D}(q)$ with boundary data zero on $\partial \mathcal{L}(q) \cap \mathcal{D}(q)$ and $P_{q, 1}$ on the rest of the boundary.

To define $\bar{U}_{q}$, we replace $\underline{L}(t)$ with $\bar{L}(t)$, where $\bar{L}$ is the reflected image of $\underline{L}$ with respect to $e_{n}$-axis.

Now for given unit vector $q$ in $\mathbb{R}^{n}$ and $r \in[1,2]$ we define

$$
\underline{U}_{q, r}(x, t):=\underline{U}_{q}(x, r t), \quad \bar{U}_{q, r}(x, t):=\bar{U}_{q}(x, r t) .
$$

Lemma 2.3. For a unit vector $q \in \mathbb{R}^{n}$ and $r \in[1,2], \underline{U}_{q, r}$ is a supersolution and $\bar{U}_{q, r}$ is a subsolution of $(P)_{\epsilon}$.

Proof. By comparing $\underline{U}_{q}(\cdot, t)$ with planar harmonic functions at each $t \in$ $[0,1]$ it follows that on its free boundary $\left|D \underline{U}_{q}\right| \leq \frac{1}{2}$. Hence the normal velocity $V$ of $\Gamma\left(\underline{U}_{q, r}\right)$ satisfies

$$
V=r \geq 1 \geq 2\left|D \underline{U}_{q, r}\right|
$$

and thus $\underline{U}_{q, r}$ is a supersolution of $(P)_{\epsilon}$.

(We mention that this is the only place that $\mathcal{D}(q)$ with skewed lateral boundaries was needed, to show that $\left|D \underline{U}_{q}\right| \leq 1 / 2$ by comparison with planar harmonic functions.)

Similarly we can show that $\left|D \bar{U}_{q}\right| \geq 2$ on $\Gamma\left(\bar{U}_{q}\right)$. Hence

$$
V=r \leq 2 \leq\left|D \bar{U}_{q, r}\right| \text { on } \Gamma\left(\bar{U}_{q, r}\right)
$$

and $\bar{U}_{q, r}$ is a subsolution of $(P)_{\epsilon}$. 
Corollary 2.4. For a unit vector $q \in \mathbb{R}^{n}$ and $r \in[1,2]$,

$$
\bar{U}_{q, r} \leq \bar{u}_{\epsilon ; q, r}, \quad \underline{u}_{\epsilon ; q, r} \leq \underline{U}_{q, r} .
$$

Lemma 2.5. For any unit vector $q \in \mathbb{R}^{n}$ and $r \in[1,2]$,

(a) $\bar{u}_{\epsilon ; q, r}$ is a subsolution of $(P)_{\epsilon}$ with $\bar{u}_{\epsilon ; q, r} \leq P_{q, r}$ in $\bar{Q}_{1}$ and $\bar{u}_{\epsilon ; q, r}=P_{q, r}$ on the parabolic boundary of $\bar{Q}_{1}$. Moreover $\bar{u}_{\epsilon ; q, r}$ is a solution of $(P)_{\epsilon}$ away from $\Gamma\left(\bar{u}_{\epsilon ; q, r}\right) \cap l_{q, r}$.

(b) $\underline{u}_{\epsilon ; q, r}$ is a supersolution of $(P)_{\epsilon}$ with $\underline{u}_{\epsilon ; q, r} \geq P_{q, r}$ in $\bar{Q}_{1}$ and $\underline{u}_{\epsilon ; q, r}=P_{q, r}$ on the parabolic boundary of $\bar{Q}_{1}$. Moreover $\underline{u}_{\epsilon ; q, r}$ is a solution of $(P)_{\epsilon}$ away from $\Gamma\left(\underline{u}_{\epsilon ; q, r}\right) \cap l_{q, r}$.

Proof. 1. We only prove the lemma for $\bar{u}_{\epsilon ; q, r}$.

2. $\bar{u}_{\epsilon ; q, r}$ is a subsolution of $(P)_{\epsilon}$ due to its definition and the stability property of viscosity solutions. $\bar{u}_{\epsilon ; q, r}=P_{q, r}$ on $\partial \mathcal{D}(q) \times[0,1]$ due to Corollary 2.4.

3. It remains to prove that $\left(\bar{u}_{\epsilon ; q, r}\right)_{*}$ is a supersolution in $Q_{1}$ away from $l_{q, r}$. Due to the definition $\bar{u}_{\epsilon ; q, r}$ is harmonic in its positive phase. Thus if our assertion is not true, then there exist a smooth function $\phi$ which touches $\left(\bar{u}_{\epsilon ; q, r}\right)_{*}$ from below at $\left(x_{0}, t_{0}\right) \in \Gamma\left(\bar{u}_{\epsilon ; q, r}\right) \cap \Omega\left(P_{q, r}\right) \cap Q_{1}$, with $|D \phi|\left(x_{0}, t_{0}\right) \neq 0$ and

$$
\min \left[\phi_{t}-g\left(\frac{x_{0}}{\epsilon}\right)|D \phi|^{2},-\Delta \phi\right]\left(x_{0}, t_{0}\right)<0 .
$$

By continuity of $g$, for sufficiently small $\delta, \gamma, r>0$, the function

$$
\Phi(x, t):=\left(\phi+\delta-\gamma\left(\left|x-x_{0}\right|^{2}+\left|t-t_{0}\right|^{2}\right)\right)_{+}
$$

is a subsolution of $(P)_{\epsilon}$ with $\Phi \leq P_{q, r}$ in $B_{r}\left(x_{0}\right) \times\left[t_{0}-r, t_{0}+r\right]$. Observe that, due to Lemma 1.9, for any $\delta, \gamma>0 \Phi>\bar{u}_{\epsilon ; q, r}$ in $B_{c}\left(x_{0}\right) \times\left[0, t_{0}\right)$, where $c$ is a small constant depending on $\delta, \gamma$.

Choose $\delta>0$ small enough such that $\Phi \leq \bar{u}_{\epsilon ; q, r}$ outside $B_{r / 2}\left(x_{0}\right) \times\left[t_{0}-\right.$ $\left.r, t_{0}+r\right]$. Now the function

$$
\Psi:=\left\{\begin{array}{l}
\max \left(\bar{u}_{\epsilon ; q, r}, \Phi\right) \text { in } B_{r}\left(x_{0}\right) \times\left[t_{0}-r, t_{0}+r\right], \\
\bar{u}_{\epsilon ; q, r} \text { otherwise }
\end{array}\right.
$$

is a strictly bigger subsolution of $(P)_{\epsilon}$ than $\bar{u}_{\epsilon ; q, r}$ and less than $P_{q, r}$ in $Q_{1}$, yielding a contradiction. 
The following corollary provides, in particular, estimates for the free boundary velocity of $\bar{u}_{\epsilon ; q, r}$ and $\underline{u}_{\epsilon ; q, r}$ in $\epsilon$-scale.

Corollary 2.6. (a) For any given unit vector $q \in \mathbb{R}^{n}$ and for any $a \in[0,1]$, there is a vector $\eta \in \mathbb{R}^{n}$ such that $a q+\eta \in \epsilon \mathbb{Z}^{n}, \eta \cdot q \geq \frac{1}{2}|\eta|$ and $\epsilon \leq|\eta|<3 \epsilon$. For this $\eta$ and for $r \in[1,2]$ the following is true:

$$
\bar{u}_{\epsilon ; q, r}\left(x+a q+\eta, t+a r^{-1}+\frac{1}{4} r^{-1} \epsilon\right) \leq \bar{u}_{\epsilon ; q, r}(x, t)
$$

and

$$
\underline{u}_{\epsilon ; q, r}\left(x+a q+\eta, t+a r^{-1}+6 r^{-1} \epsilon\right) \geq \underline{u}_{\epsilon ; q, r}(x, t) \text { in } Q_{1} .
$$

(b) For $\eta$ as given in (a) for $a=0$, we have

$$
\bar{u}_{\epsilon ; q, r}\left(x-\eta, t+6 r^{-1} \epsilon\right) \geq \bar{u}_{\epsilon ; q, r}(x, t)
$$

and

$$
\underline{u}_{\epsilon ; q, r}\left(x-\eta, t+\frac{1}{4} r^{-1} \epsilon\right) \leq \underline{u}_{\epsilon ; q, r}(x, t) \text { in } Q_{1} .
$$

Proof. 1. (a) is due to Corollary 2.4, Lemma 2.5 and the definition of $\bar{u}_{\epsilon ; q, r}$ and $\underline{u}_{\epsilon ; q, r}$.

2. By barrier argument one can check that

$$
\bar{u}_{\epsilon ; q, r}\left(x+\eta, 4 r^{-1} \epsilon\right) \geq P_{q, r}(x, 0) \text { in } \mathcal{D}(q) \cap\{x \cdot q \leq 2 \epsilon\} .
$$

the first inequality in (b) follows from above inequality, Corollary 2.4, Lemma 2.5 and Theorem 1.7. The second inequality can be checked similarly.

For a unit vector $q \in \mathbb{R}^{n}$ and $r \in[1,2]$, define

$$
\bar{A}_{\epsilon ; q, r}=\Gamma_{1}\left(\bar{u}_{\epsilon ; q, r}\right) \cap l_{q, r}(1) \cap \frac{1}{2} \mathcal{D}(q)
$$

and

$$
\underline{A}_{\epsilon ; q, r}=\Gamma_{1}\left(\underline{u}_{\epsilon ; q, r}\right) \cap l_{q, r}(1) \cap \frac{1}{2} \mathcal{D}(q)
$$

Also define

$$
\bar{r}(q)=\sup \left\{r: \bar{A}_{\epsilon ; q, r} \neq \emptyset \text { for } \epsilon \leq \epsilon_{0} \text { with some } \epsilon_{0}>0\right\} \in[1,2]
$$

and

$$
\underline{r}(q)=\inf \left\{r: \underline{A}_{\epsilon ; q, r} \neq \emptyset \text { for } \epsilon \leq \epsilon_{0} \text { with some } \epsilon_{0}>0\right\} \in[1,2] .
$$

Throughout the paper we will call $\mu=a_{1} e_{1}+\ldots a_{n} e_{n}$ a lattice vector if $a_{i} \in \mathbb{Z}$, and $\mu$ a rational vector if $a_{i} \in \mathbb{Q}$. 
Lemma 2.7. If $P_{0} \in \bar{\Omega}_{t_{0}}\left(\bar{u}_{\epsilon ; q, r}\right) \cap \frac{1}{2} \mathcal{D}(q)$, then

$$
\left\{2\left(P_{0}-\frac{r t_{0} q}{2}-\mu\right): \frac{r t_{0} q}{2}+\mu \in \epsilon \mathbb{Z}^{n}, q \cdot \mu \geq 0\right\} \cap \mathcal{D}(q) \subset \bar{\Omega}_{t_{0}}\left(\bar{u}_{2 \epsilon ; q, r}\right) .
$$

Note that $2 P_{0}-r t_{0} q \in l_{q, r}\left(t_{0}\right)$ if $P_{0} \in l_{q, r}\left(t_{0}\right)$.

Proof. We compare

$$
u_{1}(x, t):=2 \bar{u}_{\epsilon_{0} ; q, r}\left(\frac{x+r t_{0} q+2 \mu}{2}, \frac{t+t_{0}}{2}\right)
$$

and

$$
u_{2}(x, t):=\bar{u}_{2 \epsilon_{0} ; q, r}(x, t)
$$

in $Q_{1}$. Since $u_{1}$ is a subsolution of $(P)_{2 \epsilon_{0}}$ with $u_{1} \leq P_{q, r}$ in $Q_{1}$, by definition of $u_{2}$ we have $u_{1} \leq u_{2}$ in $Q_{1}$ and the conclusion follows.

Below we state the corresponding lemma for $\bar{u}$. The proof is parallel to the above lemma.

Lemma 2.8. If $P_{0}$ is in $\Omega_{t_{0}}\left(\underline{u}_{\epsilon ; q, r}\right)$, then

$$
\left\{\frac{P_{0}+r t_{0} q+\mu}{2}: r t_{0} q+\mu \in \epsilon \mathbb{Z}^{n}, q \cdot \mu \geq 0\right\} \cap \frac{1}{2} \mathcal{D}(q) \subset \Omega_{t_{0}}\left(\underline{u}_{\epsilon / 2 ; q, r}\right) .
$$

The following lemma plays an important role in the rest of the paper.

Lemma 2.9. Fix a unit vector $q \in \mathbb{R}^{n}$ and $r \in[1,2]$.

(a) Suppose $d\left(\Gamma\left(\underline{u}_{\epsilon ; q, r}\right), l_{q, r}\right)<1 / 100$. Then there exists a dimensional constant $M>0$ such that, for any $x_{0} \in \Gamma_{t_{0}}\left(\underline{u}_{\epsilon ; q, r}\right)$,

$$
d\left(\Gamma_{t_{0}}\left(\underline{u}_{\epsilon / 2 ; q, r}\right) \cap \frac{1}{2} \mathcal{D}(q), l_{q, r}\left(t_{0}\right)\right)>d\left(\frac{x_{0}+r t_{0} q}{2}, l_{q, r}\left(t_{0}\right)\right)-\frac{M}{2} \epsilon .
$$

In particular if $\underline{A}_{\epsilon / 2 ; q, r}$ is nonempty then

$$
d\left(x, l_{q, r}(t)\right) \leq M \epsilon \text { for } x \in \Gamma_{t}\left(\underline{u}_{\epsilon ; q, r}\right), 0 \leq t \leq 1 .
$$

(b) Suppose $d\left(\Gamma\left(\bar{u}_{\epsilon ; q, r}\right), l_{q, r}\right)<1 / 100$. Then there exists a dimensional constant $M>0$ such that, for any $x_{0} \in \Gamma_{t_{0}}\left(\bar{u}_{\epsilon ; q, r}\right) \cap \frac{1}{2} \mathcal{D}(q)$,

$$
d\left(\Gamma_{t_{0}}\left(\bar{u}_{2 \epsilon ; q, r}\right), l_{q, r}\left(t_{0}\right)\right)>d\left(2 x_{0}-r t_{0} q, l_{q, r}\left(t_{0}\right)\right)-\frac{M}{2} \epsilon
$$

In particular if $\bar{A}_{2 \epsilon ; q, r}$ is nonempty then

$$
d\left(x, l_{q, r}(t)\right) \leq M \epsilon \text { for } x \in \Gamma_{t}\left(\bar{u}_{\epsilon ; q, r}\right) \cap \frac{1}{2} \mathcal{D}(q), 0 \leq t \leq 1 .
$$


Proof. 1. We only prove (a), a parallel argument holds for (b). Observe that once (2.1) is proved the second assertion in (a) follows, for $t=1$ from (2.1) and for $0 \leq t<1$ from Corollary 2.6.

2. For simplicity we drop $q, r$ in the notation of $\underline{u}_{\epsilon ; q, r}$ in the proof.

3. Let $x_{0}$ to be the furthest point of $\Gamma_{t_{0}}\left(\underline{u}_{\epsilon}\right)$ from $l_{q, r}\left(t_{0}\right)$ in $\mathcal{D}(q)$. We may assume that

$$
d\left(x_{0}, l_{q, r}\left(t_{0}\right)\right) \geq M \epsilon
$$

since otherwise the lemma is proved. By our hypothesis, there exists

$$
\underline{u}_{\epsilon}\left(\cdot, t_{0}\right)=0 \text { in } B_{6 e}\left(x_{0}+6 \epsilon q\right) .
$$

Due to (2.4) and Lemma 1.9, $\left(\underline{u}_{\epsilon}\right)^{*}\left(\cdot, t_{0}\right) \equiv 0$ in $B_{3 \epsilon}\left(x_{0}+6 \epsilon q\right)$. We claim that there exists a dimensional constant $c_{0}$ such that

$$
\sup _{\left\{\left|y-x_{0}\right| \leq 4 \epsilon\right\}} \underline{u}_{\epsilon}\left(y, t_{0}\right) \geq c_{0} \epsilon .
$$

Due to Corollary 2.6,

$$
\underline{u}_{\epsilon}\left(\cdot, t_{0}-16 \epsilon\right) \equiv 0 \text { in } B_{2 \epsilon}\left(x_{0}\right) .
$$

For sufficiently small $c_{0}>0$, consider the function $\varphi(x, t)$ defined in $\Sigma:=\bar{B}_{4 \epsilon}\left(x_{0}\right) \times\left[t_{0}-16 \epsilon, t_{0}\right)$ such that

$$
\left\{\begin{array}{lll}
-\Delta \varphi(\cdot, t) \quad \text { in } & B_{4 \epsilon}\left(x_{0}\right)-B_{r(t) \epsilon}\left(x_{0}\right) ; \\
\varphi=c_{0} \epsilon & \text { on } & \partial B_{4 \epsilon}\left(x_{0}\right) \times\left[t_{0}-16 \epsilon, t_{0}\right] ; \\
\varphi(\cdot, t)=0 \quad \text { in } & B_{r(t) \epsilon}\left(x_{0}\right),
\end{array}\right.
$$

where $r(t)=2-\frac{1}{16}\left(t-t_{0}+16 \epsilon\right)$, is a supersolution of $(P)_{\epsilon}$.

Observe that $\underline{u}_{\epsilon}$ is a viscosity solution of $(P)_{\epsilon}$ in $\Sigma$ due to (2.3) and Lemma 2.5. If (2.5) is not true, then we apply Theorem 1.7 to $\varphi$ and $\left(\underline{u}_{\epsilon}\right)^{*}$ in $B_{4 \epsilon}\left(x_{0}\right) \times\left[t_{0}-16 \epsilon, t_{0}\right)$, to show that $\Gamma\left(\underline{u}_{\epsilon}\right)$ cannot reach $x_{0}$ by time $t_{0}$, a contradiction. Thus there exists $y_{0} \in B_{4 \epsilon}\left(x_{0}\right)$ such that $\underline{u}_{\epsilon}\left(y_{0}, t_{0}\right) \geq c_{0} \epsilon$. By lower semi-continuity of $\underline{u}_{\epsilon}$ there is a small spatial ball $B_{\delta}\left(y_{0}\right)$ where $\underline{u}_{\epsilon}>0$.

4. We next claim that there exists a dimensional constant $M>0$ such that

$$
\underline{u}_{\epsilon}\left(\cdot, t_{0}+3 M \epsilon\right)>0 \text { in } B_{3 \epsilon}\left(x_{0}\right) .
$$


If $\delta>7 \epsilon$ we are done. If not, by Harnack inequality for harmonic functions and by the fact that $\underline{u}_{\epsilon}$ is increasing in time, there exists a dimensional constant $c_{1}>0$ such that

$$
\underline{u}_{\epsilon}(\cdot, t) \geq c_{1} \epsilon \text { in } B_{r}\left(y_{0}\right) \text { for } t \geq t_{0}
$$

if $B_{2 r}\left(y_{0}\right) \in \Omega_{t}\left(\underline{u}_{\epsilon}\right)$.

Thus if we choose a sufficiently large dimensional constant $M>0$, then a radially symmetric harmonic function $\phi(\cdot, t)$ in the ring domain

$$
B_{M^{-1} t+\delta}\left(y_{0}\right)-\frac{1}{2} B_{M^{-1} t+\delta}\left(y_{0}\right)
$$

with fixed boundary data $c_{1} \epsilon$ on the inner ring and zero on the outer ring is a subsolution of $(P)_{\epsilon}$ in

$$
\Sigma:=\left(\mathbb{R}^{n}-\frac{1}{2} B_{M^{-1} t+\delta}\left(y_{0}\right)\right) \times[0,3 M \epsilon]
$$

with $\phi(x, t) \leq \underline{u}_{\epsilon}\left(x, t+t_{0}\right)$ on the parabolic boundary of $\Sigma$.

5. It follows that at $\underline{u}_{\epsilon}\left(\cdot, t_{0}+3 M \epsilon\right)>0$ in $B_{3 \epsilon}\left(x_{0}\right)$. By Lemma 2.7 it follows that $\underline{u}_{\epsilon / 2 ; q, r}\left(\cdot, t_{0}+3 M \epsilon\right)>0$ in the set

$$
\left\{y: d\left(y, l_{p, q}\left(t_{0}+3 M \epsilon\right)\right) \leq d\left(\frac{x_{0}+r t_{0} q}{2}, l_{p, q}\left(t_{1}\right)\right)\right\},
$$

proving the lemma.

For the next section, where we consider limits of the solutions of $(P)_{\epsilon}$, here we prove that $\underline{u}_{\epsilon ; q, r}\left(\bar{u}_{\epsilon ; q, r}\right)$ is 'non-degenerate' on their free boundaries.

Lemma 2.10. (a) Suppose $1 \leq r \leq 2$. Then there exists a dimensional constant $c=c(n)$ such that for any $0<h \leq d$ and for any $\left(x_{0}, t_{0}\right) \in$ $\Gamma\left(\bar{u}_{\epsilon ; q, r}\right) \cap\left(\frac{1}{2} \mathcal{D}(q) \times[0,1]\right)$,

$$
\sup _{\left\{\left|x_{0}-y\right|<h\right\}} \bar{u}_{\epsilon ; q, r}\left(y, t_{0}\right) \geq c h^{2} /(d+h) .
$$

where $d=d\left(l_{q, r}\left(t_{0}\right), x_{0}\right)$.

(b) Suppose $\underline{r}(q) \leq r \leq 2$. Then there exists a dimensional constant $c=c(n)$ such that for $h \leq M \epsilon$ where $M$ is given as in Lemma 2.9 and for any $\left(x_{0}, t_{0}\right) \in \Gamma\left(\underline{u}_{\epsilon ; q, r}\right) \cap\left(\frac{1}{2} \mathcal{D}(q) \times[0,1]\right)$, we have

$$
\sup _{\left\{\left|x_{0}-y\right|<h\right\}} \underline{u}_{\epsilon ; q, r}\left(y, t_{0}\right) \geq c h^{2} / M \epsilon .
$$


Proof. We first prove the lemma for $\bar{u}_{\epsilon ; q, r}$. Due to the definition of $d$, for $h<d$,

$$
\bar{u}_{\epsilon ; q, r}\left(\cdot, t_{0}-(d+h)\right) \equiv 0 \text { in } B_{h}\left(x_{0}\right) .
$$

If (a) is not true with sufficiently small $c>0$, then a barrier argument with a radially symmetric function, as in the proof of Lemma 2.9 , yields that

$$
\bar{u}_{\epsilon ; q, r}\left(\cdot, t_{0}\right) \equiv 0 \text { in } B_{h / 2}\left(x_{0}\right),
$$

which is a contradiction.

To prove (b), first suppose that $d\left(B_{h / 2}\left(x_{0}\right), l_{q, r}\left(t_{0}\right)\right)>0$. Since $\underline{r}(q) \leq r$, $\Gamma\left(\underline{u}_{\epsilon ; q, r}\right) \cap\left(\frac{1}{2} \mathcal{D}(q) \times[0,1]\right)$ is within $M \epsilon$-distance of $l_{q, r}$ due to Lemma 2.9 . Thus

$$
\underline{u}_{\epsilon ; q, r}\left(\cdot, t_{0}-(M \epsilon+h)\right) \equiv 0 \text { in } B_{h}\left(x_{0}\right) .
$$

Suppose for some $0<h \leq d, \underline{u}_{\epsilon ; q, r} \leq \frac{c h^{2}}{M \epsilon}$ in $B_{h}\left(x_{0}\right)$. If $c$ is sufficiently small, again a barrier argument with a radially symmetric supersolution of $(P)_{\epsilon}$, using the fact that $\underline{u}_{\epsilon ; q, r}$ is a solution in $B_{h / 2}\left(x_{0}, t_{0}\right)$ leads to a contradiction.

If $d\left(x_{0}, l_{q, r}\left(t_{0}\right)\right) \leq h / 2$ then the lemma holds due to the fact that $\underline{u}_{\epsilon ; q, r}\left(\cdot, t_{0}\right) \geq$ $P_{q, r}\left(\cdot, t_{0}\right)$.

\section{Uniqueness of the limiting velocity}

Let us define

$$
\bar{u}_{\epsilon ; q, r}^{\infty}(x, t):=\left(\limsup _{n \rightarrow \infty} \bar{u}_{\epsilon ; q, r}^{n}\right)^{*}
$$

where $\bar{u}_{\epsilon ; q, r}^{n}(x, t):=n \bar{u}_{\epsilon / n ; q, r}(x / n, t / n)$. Let us also define

$$
\underline{u}_{\epsilon ; q, r}^{\infty}:=\left(\liminf _{n \rightarrow \infty} \underline{u}_{\epsilon ; q, r}^{n}\right)_{*}
$$

where $\underline{u}_{\epsilon ; q, r}^{n}(x, t):=n \underline{u}_{\epsilon / n ; q, r}(x / n, t / n)$.

Lemma 3.1. (a) $\bar{u}_{\epsilon ; q, r}^{\infty}\left(\underline{u}_{\epsilon ; q, r}^{\infty}\right)$ is a sub(super) solution of $(P)_{\epsilon}$, less (bigger) than $P_{q, r}$, with initial data $P_{q, r}(x, 0)$ in $\mathbb{R}^{n} \times[0, \infty)$.

(b) For $r \leq \bar{r}(q)$

$$
\bar{u}_{\epsilon ; q, r}^{\infty}(x+\mu, t)=\bar{u}_{\epsilon ; q, r}^{\infty}(x, t)
$$

for any lattice vector $\mu$ orthogonal to $q$.

(The same equality holds for $\underline{u}_{\epsilon ; q, r}$ holds for $r \geq \underline{r}(q)$.) 
(c) for any lattice vector $\mu$,

$$
\bar{u}_{\epsilon ; q, r}^{\infty}\left(x+\epsilon \mu, t+r^{-1} \epsilon \mu \cdot q\right) \leq \bar{u}_{\epsilon ; q, r}^{\infty}(x, t)
$$

and

$$
\underline{u}_{\epsilon ; q, r}^{\infty}\left(x+\epsilon \mu, t+r^{-1} \epsilon \mu \cdot q\right) \geq \underline{u}_{\epsilon ; q, r}^{\infty}(x, t) .
$$

(d) For $r \leq \bar{r}(q), \bar{u}_{\epsilon ; q, r}^{\infty}$ has 'almost flat' free boundary:

$$
d\left(\Gamma_{t}\left(\bar{u}_{\epsilon ; q, r}^{\infty}\right), l_{q, r}(t)\right) \leq M \epsilon \text { for } 0 \leq t \leq 1 .
$$

(The same inequality for $\underline{u}_{\epsilon ; q, r}^{\infty}$ holds if $r \geq \underline{r}(q)$.)

Proof. 1. We will only prove the lemma for $\bar{u}_{\epsilon ; q, r}^{\infty}$.

2. Note that $\bar{u}_{\epsilon ; q, r}^{n}$ is the maximal subsolution which is smaller than $P_{q, r}$ in $Q_{n}:=n \mathcal{D}(q) \times[0, n]$ with boundary data equal to $P_{q, r}$ on the parabolic boundary of $Q_{n}$. Therefore $\bar{u}_{\epsilon ; q, r}^{n}$ is decreasing in $n$. Moreover $\bar{u}_{\epsilon ; q, r}^{n}(\cdot, t) \geq$ $P_{q, r}(x, 0)$ for $t \geq 0$, and therefore we conclude that $\bar{u}_{\epsilon ; q, r}^{\infty}(x, 0)=P_{q, r}(0)$. Since $r \leq \bar{r}(q)$, by Lemma 2.7 there exists a dimensional constant $M>0$ such that

$$
d\left(x, l_{q, r}(t)\right)<M \epsilon \text { for any } x \in \Gamma_{t}\left(\bar{u}_{\epsilon ; q, r}^{n}\right)
$$

for sufficiently large $n$. It then follows from (3.1) and Lemma 2.10 and for any $\left(x_{0}, t_{0}\right) \in \Gamma\left(\bar{u}_{\epsilon ; q, r}^{n}\right)$

$$
\sup _{B_{h}\left(x_{0}\right)} \bar{u}_{\epsilon}^{n} \geq c \frac{h^{2}}{M \epsilon+h},
$$

where $c$ is a dimensional constant. Thus

$$
\bar{\Omega}\left(\bar{u}_{\epsilon ; q, r}^{\infty}\right)=\limsup _{\epsilon \rightarrow 0} \bar{\Omega}\left(\bar{u}_{\epsilon ; q, r}^{n}\right) .
$$

Now standard viscosity solutions argument will prove that $\bar{u}_{\epsilon ; q, r}^{\infty}$ is a viscosity subsolution of $(P)_{\epsilon}$.

3. Suppose $\mu \in \mathbb{Z}^{n}$ with $\mu \cdot q=0$. Observe that, for any $n \geq N \geq \epsilon|\mu|$,

$$
\bar{u}_{\epsilon ; q, r}^{n+N}(x+\epsilon \mu, t) \leq \bar{u}_{\epsilon ; q, r}^{n}(x, t) \leq \bar{u}_{\epsilon ; q, r}^{n-N}(x+\epsilon \mu, t) \text { in } Q_{n},
$$

Hence taking $n \rightarrow \infty$ it follows that

$$
\bar{u}_{\epsilon ; q, r}^{\infty}(x+\epsilon \mu, t)=\bar{u}_{\epsilon ; q, r}^{\infty}(x, t) .
$$

4. (c) follows from the fact that, for any $\mu \in \mathbb{Z}^{n}$ and $N \geq|\mu|$,

$$
\bar{u}_{\epsilon ; q, r}^{n+N}\left(x+\epsilon \mu, t+r^{-1} \epsilon \mu \cdot q\right) \leq \bar{u}_{\epsilon ; q, r}^{n}(x, t) .
$$

5. (d) follows from (3.1) and (3.3). 
Lemma 3.2. $\bar{r}(q) \leq \underline{r}(q)$ for any rational unit vector $q \in \mathbb{R}^{n}$.

Proof. Suppose $\bar{r}(q)-\underline{r}(q)=\sigma>0$. Choose $r_{3}=\underline{r}(q)+\sigma / 3$ and $r_{4}=$ $\underline{r}(q)+2 \sigma / 3=\bar{r}(q)-\sigma / 3$. Consider small positive constants $0<\delta<<\gamma$, $\gamma<\sigma / 20$ and a lattice vector $\eta$ such that $|\eta|<2, q \cdot \eta \leq-1 / 2$. Define

$$
U_{1}(x, t):=\sup _{d((y, s),(x, t))<\delta \epsilon} u_{1}(x, t)
$$

where

$$
u_{1}(x, t):=(1-\gamma) \bar{u}_{\epsilon ; q, r_{4}}^{\infty}(x,(1-\sigma / 20) t)
$$

and

$$
U_{2}(x, t):=\inf _{(d((y, s),(x, t))<\delta \epsilon} u_{2}(x, t)
$$

where

$$
u_{2}(x, t):=\underline{u}_{\epsilon ; q, r_{3}}^{\infty}(x+\epsilon \eta,(1+\sigma / 20) t) .
$$

Observe that $U_{1}$ and $U_{2}$ are respectively sub- and supersolution of $(P)_{\epsilon}$ in $\mathbb{R}^{n} \times[0,1]$ if $\delta$ is chosen small enough with respect to $\sigma$ and the continuity mode of $g$.

We compare $U_{1}$ and $U_{2}$ in $Q_{1}$. By the choice of $\delta, \gamma, \sigma$ we have $U_{1} \leq$ $P_{q, r} \leq U_{2}$ on the set $\{x: x \cdot q=-1\} \times[0, \gamma / \sigma]$.

Note that $l_{q, r_{4}(1-\sigma / 20)}$ propagates faster than $l_{q, r_{3}(1+\sigma / 20)}$ by more than $\sigma / 10$. Moreover by definition and Lemma 3.1 (d) $\Gamma\left(U_{1}\right)$ and $\Gamma\left(U_{2}\right)$ are respectively within $(M+2+\delta) \epsilon<2 M \epsilon$-distance of $l_{q, r_{3}}$ and $l_{q, r_{4}}$.

Since the free boundaries cannot jump in time (Lemma 1.9) $\Gamma\left(U_{2}\right)$ will contact $\Gamma\left(U_{1}\right)$ for the first time in $\bar{Q}_{1}$ at a point $\left(x_{0}, t_{0}\right), t_{0} \in(0,40 M \epsilon / \sigma)$. Let us choose $\gamma=40 M \epsilon$ and $\epsilon \leq \frac{\sigma}{1000 M}$ so that

$$
U_{1} \leq U_{2} \text { on }\{x: x \cdot q=-1\} \times\left[0, t_{0}\right] .
$$

Due to the periodicity of $u_{1}$ and $u_{2}$ (Lemma 3.1) and the maximum principle of harmonic functions, it follows that $U_{1} \leq U_{2}$ in $\mathcal{D}(q) \times\left[0, t_{0}\right]$. Since $q$ is rational, by Lemma 3.1 (b) there are other first contact points in the interior of $\mathcal{D}(q)$. Now one can argue as in the proof of Theorem 2.2 of [K1] to derive a contradiction.

The argument in the proof of Lemma 3.2, while simple, does not apply to the cases with non-rational vectors $q \in \mathbb{R}^{n}$ due to the loss of periodicity of $u_{1}$ and $u_{2}$ on the free boundary. Hence we will apply a more careful argument, based on the property of rational and irrational numbers, for the general proof. 
Lemma 3.3. Suppose $n_{1}, n_{2} \geq 1$ are integers, prime to each other. Then there exist integers $0<k_{1}<n_{2}, 0<k_{2} \leq n_{1}$ such that

$$
\left|k_{1} n_{1}-k_{2} n_{2}\right|=1 \text {. }
$$

Proof. Let $n_{1}<n_{2}$. If our claim is not true, then the set

$$
S=\left\{0 \leq k_{1}<n_{2}, 0 \leq k_{2}<n_{1}: k_{1} n_{1}-k_{2} n_{2}\right\}
$$

are all apart by at least 2 . Since $n_{1}$ and $n_{2}$ are prime to each other, the elements in $S$ are all distinct and thus

$$
|S|=n_{1} n_{2} \text { and } S \subset I:=\left[-n_{1} n_{2}+n_{2}, n_{1} n_{2}-n_{1}\right],
$$

where $I$ contains $2 n_{1} n_{2}-n_{1}-n_{2}+1<2 n_{1} n_{2}-1$ integers. Since the elements of $S$ are all apart by 2, a contradiction follows.

We will next prove that, for a rational unit vector $q$, if $r$ is bigger than $\bar{r}(q)$ then for sufficiently small $\epsilon$ the free boundary of $\bar{u}_{\epsilon ; q, r}$ falls behind $l_{q, r}$ by a positive distance after a positive amount of time. The estimate on this distance, the amount of time after which the free boundary falls behind, and the size of $\epsilon$ obtained in the following lemma is essential to the analysis later in the section.

Proposition 3.4. Suppose $q$ is a unit vector in $\mathbb{R}^{n}$,

$$
q=m\left(e_{1}+\frac{a_{2}}{N_{2}} e_{2}+\ldots+\frac{a_{n}}{N_{n}} e_{n}\right),
$$

where $1 / n \leq|m| \leq 1$ and $a_{2}, N_{2}$ are relatively prime integers and $a_{i}, N_{i} \in \mathbb{Z}$ with $0 \leq a_{i} / N_{i} \leq 1$ for $i=2, \ldots, n$. Let $N=\max N_{i}$.

Suppose $r=r_{0}+C(n) \gamma \leq 2 r_{0}, r_{0}:=\bar{r}(q)$ where $C(n)$ is a sufficiently large dimensional constant. If $1 / N<\gamma^{2}$ then for $0<\epsilon<\epsilon_{0}=\frac{\gamma^{2}}{8 n M N}$,

$$
d\left(\Gamma_{t}\left(\bar{u}_{\epsilon ; q, r}\right), l_{q, r}(t) \cap B_{1 / 4}(0)\right)>M \epsilon_{0}
$$

for $\frac{M \epsilon_{0}}{\gamma} \leq t \leq 1$, where $M$ is the dimensional constant given in Lemma 2.9.

Proof. 1. Without loss of generality we may assume that $N=N_{2}$. Let us denote

$$
\eta_{i}:=e_{i}-\frac{a_{i}}{N_{i}} e_{1}, i=2, \ldots, n
$$




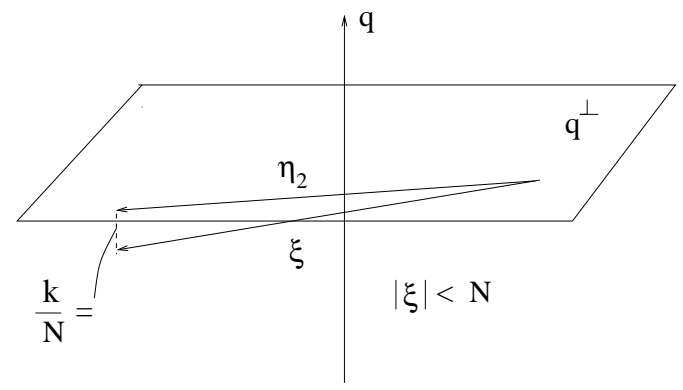

Figure 5.

Note that $\left\{\eta_{i}\right\}_{i=2, . ., n}$ is a basis for the hyperplane $\{x: x \cdot q=0\}$ and $\left|\eta_{i} \cdot \eta_{j}\right| \leq \frac{1}{2}\left|\eta_{i}\right|\left|\eta_{j}\right|$.

By the previous lemma there exist integers $k_{1}, k_{2} \in[-N, N]$ such that for any $c \in \mathbb{R}$

$$
q \cdot\left(c \eta_{2}-\left(k_{1} e_{2}+k_{2} e_{1}\right)\right)=m\left(k_{1} a_{2} / N-k_{2}\right)=-m / N<\gamma .
$$

On the other hand $N \eta_{2}$ is a lattice vector in $e_{2}-e_{1}$ plane. Hence for any integer $k$ there exists a lattice vector $\xi$ in $e_{2}-e_{1}$ plane such that

$$
0 \leq|\xi| \leq N, \quad q \cdot \xi=-k m / N
$$

(see Figure 5.)

We choose $k$ such that

$$
(k-1) m / N<\gamma \leq k m / N \text {, i.e. } k m / N \in(\gamma, 2 \gamma) .
$$

2. Next consider the domain $\mathcal{O}:=\Pi \cap\left[0,2 M \epsilon_{0} / \gamma\right]$, where $M$ is the constant given in Lemma 2.9 and

$$
\Pi:=\left\{(x, t):|x| \leq 1 / 2+\frac{(n+1) N}{\gamma} t, 0 \leq t \leq 2 M \epsilon_{0} / \gamma\right\} .
$$

Observe that $\mathcal{O} \subset Q_{1}$. Let us define $u_{1}:=\breve{u}_{\epsilon ; q, r}$, where $\breve{u}_{\epsilon ; q, r}$ is the maximal subsolution below $P_{q, r}$, defined the same as $\bar{u}_{\epsilon ; q, r}$, in the domain $\mathcal{O}$ instead of $Q_{1}$. A parallel argument as in Lemma 2.3 yields that $u_{1}=P_{q, r}$ on the parabolic boundary of $\mathcal{O}$. Note that $\bar{u}_{\epsilon ; q, r} \leq u_{1}$ since $\mathcal{O} \subset Q_{1}$. Moreover using the definition of $u_{1}$ one can check that $\Omega\left(u_{1}\right)$ expands in time and

$$
u_{1}(x, s) \leq C u_{1}(x, t) \text { for } 0 \leq s<t \leq 2 M \epsilon_{0} / \gamma \text { and } x \in B_{1 / 4}(0) .
$$

for a dimensional constant $C>0$ (note that $u_{1}$ may not increase in time since the lateral boundary of $\Pi$ changes in time.) 
It follows from (3.4), the definition of $\Pi, u_{1}$, and Theorem 1.7 that

$$
u_{1}\left(x+(\xi+\mu) \epsilon, t+\frac{k m}{N} r^{-1} \epsilon\right) \leq u_{1}(x, t),
$$

in $B_{1 / 4}(0)$, where $\mu$ is any lattice vector orthogonal to $q$ such that $|\mu| \leq n N$.

Let

$$
\alpha:=\frac{r-r_{0}-2 \gamma}{r_{0}+r}=\frac{(C(n)-2) \gamma}{r_{0}+r}
$$

and

$$
u_{2}(x, t):=\left(1+\frac{\alpha}{4}\right) \underline{u}_{\epsilon ; q, r_{0}}^{\infty}\left(x,(1+\alpha) t+M \epsilon+C_{1} \gamma \epsilon\right)
$$

where $C_{1}>0$ is a dimensional constant to be chosen later.

Parallel argument as in the case of $u_{1}$ yields that

$$
u_{2}\left(x+(\xi+\mu) \epsilon, t+\frac{k m}{N}(1+\alpha)^{-1} r_{0}^{-1} \epsilon\right) \geq u_{2}(x, t) .
$$

in $B_{1 / 4}(0)$, where $\mu$ is as given in (3.6).

3. Finally, set

$$
\tilde{u}_{1}(x, t):=\sup _{y \in B_{\gamma \epsilon}(x)} u_{1}(y,(1-\alpha) t) ; \quad \tilde{u}_{2}(x, t):=\inf _{y \in B_{C_{1} \gamma \epsilon}(x)} u_{2}(y, t) .
$$

Note that $\tilde{u}_{1}$ and $\tilde{u}_{2}$ are respectively a sub- and supersolution of $(P)_{\epsilon}$ if $C(n)$ is large with respect to $C_{1}$. Our goal is to prove that

$$
\tilde{u}_{1} \leq \tilde{u}_{2} \text { in } \Sigma:=\bar{B}_{1 / 4}(0) \times\left[0,2 M \epsilon_{0} / \gamma\right]
$$

if $C_{1}$ and $C(n)$ is sufficiently large.

Due to Lemma 3.1, $\Gamma\left(u_{2}\right)$ stays within the $M \epsilon$-strip of $l_{q, r}(t)$. This and the fact that $(1-\alpha) r-(1+\alpha) r_{0}=2 \gamma$ and $\bar{u}_{\epsilon ; q, r} \leq u_{1}$ yields our theorem for $\frac{M \epsilon_{0}}{\gamma} \leq t \leq \frac{2 M \epsilon_{0}}{\gamma}$ once (3.10) is proved. For $M \epsilon_{0} / \gamma \leq t \leq 1$, the theorem holds due to Corollary 2.6, (a) for $\bar{u}_{\epsilon ; q, r}$.

4. Suppose that $\tilde{u}_{1}$ contacts $\tilde{u}_{2}$ from below at $\left(x_{0}, t_{0}\right)$ for the first time in $\Sigma$. Let us define

$$
\mathcal{S}:=\left\{y \in B_{1 / 2}(0):\left|\left(y-x_{0}\right) \cdot v\right| \leq N \epsilon \text { for any } v \text { orthogonal to } q .\right\}
$$

By our definition of $q$, for any point $x \in \mathcal{S}$, one can find a lattice vector $\mu$ orthogonal to $q$ such that

$$
(n-1) N \leq|\mu| \leq n N, \quad x+(\xi+\mu) \epsilon \in B_{1 / 4}(0) .
$$


(For example $\mu$ can be chosen as $b N_{i} \eta_{i}$, where $i$ is chosen such that $e_{i} \cdot \frac{x}{|x|} \leq$ $-1 / n$ and $b \in \mathbb{Z}^{+}$satisfies $b N_{i} \in\left[n N-N_{i}, n N\right]$.

Due to (3.6) and (3.9), we have

$$
\tilde{u}_{1}\left(x, t-2 \gamma^{2} \epsilon\right) \leq \tilde{u}_{2}(x, t) \text { in } \mathcal{S} \times\left[t_{0}-M \epsilon, t_{0}\right]
$$

since

$$
\begin{aligned}
\tilde{u}_{1}\left(x, t-2 \gamma^{2} \epsilon\right) & \leq \tilde{u}_{1}\left(x+(\xi+\mu) \epsilon, t-2 \gamma^{2} \epsilon-\frac{k m}{N} \epsilon(1-\alpha)^{-1} r^{-1}\right) \\
& \leq \tilde{u}_{2}\left(x+(\xi+\mu) \epsilon, t-2 \gamma^{2} \epsilon-\frac{k m}{N} \epsilon(1-\alpha)^{-1} r^{-1}\right) \\
& \leq \tilde{u}_{2}\left(x, t-2 \gamma^{2} \epsilon+\frac{k m}{N} \epsilon\left((1+\alpha)^{-1} r_{0}^{-1}-(1-\alpha)^{-1} r^{-1}\right)\right. \\
& \leq \tilde{u}_{2}(x, t),
\end{aligned}
$$

where the second inequality is due to the fact $\tilde{u}_{1} \leq \tilde{u}_{2}$ in $B_{1 / 4}(0) \times\left[t_{0}-\right.$ $\left.M \epsilon, t_{0}\right)$.

5.

Lemma 3.5. If $C_{1}=C_{1}(n)$ in (3.8) is sufficiently large, then

$$
\tilde{u}_{1}(x, t) \leq \inf _{y \in B_{2 \gamma \epsilon}(x)} u_{2}(y, t)
$$

on $\Gamma\left(\tilde{u}_{1}\right) \cap\left(\mathcal{S} \times\left[t_{0}-\epsilon, t_{0}\right]\right)$ and

$$
\tilde{u}_{1}(x, t) \leq \inf _{y \in B_{\gamma \epsilon}(x)} u_{2}(y, t)
$$

in $\Omega\left(\tilde{u}_{1}\right) \cap\left(B_{3 M \epsilon}\left(x_{0}\right) \times\left[t_{0}-3 M \epsilon, t_{0}\right]\right)$.

\section{Proof of Lemma 3.5}

1. To show (3.12), we first note that if $(y, s) \in \Gamma\left(u_{1}\right)$ with $y \in B_{1 / 2}(0)$, then $u_{1}(\cdot, s) \leq 2 \epsilon$ on $B_{c \epsilon}(y)$ if $c=c(n)$ is small enough: otherwise (3.5), a barrier argument and the Harnack inequality for harmonic functions shows that it violates the fact

$$
u_{1}(x+\epsilon \mu, t+\epsilon) \leq u_{1}(x, t) \text { in } \mathcal{O}
$$

for any lattice vector $\mu$ such that $\mu \cdot q \geq 1$. Hence for $0 \leq t \leq 2 M \epsilon_{0} / \gamma$,

$$
\tilde{u}_{1}(\cdot, t) \leq 2 \epsilon \text { in }\left\{y: d\left(y,\left\{\tilde{u}_{1}(\cdot, t)=0\right\}\right) \leq c(n) \epsilon\right\} \cap B_{1 / 2}(0) .
$$


By definition of $\tilde{u}_{2}$ and by $(3.11)$, for any $\left(z_{0}, \tau_{0}\right) \in \Gamma\left(\tilde{u}_{2}\right) \cap\left(\mathcal{S} \times\left[t_{0}-\epsilon, t_{0}\right]\right)$ there is a spatial ball $B_{1}:=B_{C_{1} \epsilon \epsilon}\left(z_{1}\right), z_{0} \in \partial B_{1}$, such that

$$
B_{1} \in\left\{\tilde{u}_{2}\left(\cdot, \tau_{0}\right)=0\right\} \cap\left\{\tilde{u}_{1}\left(\cdot, \tau_{0}-2 \gamma^{2} \epsilon\right)=0\right\}
$$

Consider a function $\varphi$ defined in the domain $\mathcal{C}=(1+\gamma \epsilon) B_{1} \times\left[0,2 \gamma^{2} \epsilon\right]$ such that

$$
\left\{\begin{array}{lll}
-\Delta \varphi(\cdot, t)=0 & \text { in } & (1+\gamma \epsilon) B_{1}-\left(1-(\gamma \epsilon)^{-1} t\right) B_{1} ; \\
\varphi(\cdot, t)=2 \epsilon & \text { on } & \partial(1+\gamma \epsilon) B_{1} ; \\
\varphi(\cdot, t)=0 & \text { outside } & \partial\left(1-(\gamma \epsilon)^{-1} t\right) B_{1} .
\end{array}\right.
$$

If $C_{1}=C_{1}(n)$ is large enough and $\gamma$ is sufficiently small so that $C_{1} \gamma(1+\gamma \epsilon) \leq$ $c(n)$ then $\varphi$ is a supersolution of $(P)_{\epsilon}$ in $\mathcal{C}$. It follows from (3.14) and Theorem 1.7 that $\tilde{u}_{1}(x, t) \leq \varphi\left(x, t-\tau_{0}+2 \gamma^{2} \epsilon\right)$ in $\mathcal{C}$, which yields $(3.12)$.

2. We claim

$$
\inf _{y \in B_{\gamma \epsilon}(x)} u_{2}(y, t) \geq C_{2} \gamma^{2} \epsilon \text { on } \Gamma\left(\tilde{u}_{1}\right)
$$

in $\mathcal{S} \times\left[t_{0}-\epsilon, t_{0}\right]$ with a dimensional constant $C_{2}$.

Due to $(3.12)$, for $\left(z_{0}, \tau_{0}\right) \in \Gamma\left(\tilde{u}_{1}\right) \cap \mathcal{S}$,

$$
B_{2 \gamma \epsilon}\left(z_{0}\right) \subset \Omega_{\tau_{0}}\left(u_{2}\right) .
$$

Now suppose that (3.15) is not true at $\left(z_{0}, t_{0}\right) \in \Gamma\left(\tilde{u}_{1}\right)$. Then due to (3.16), the harnack inequality for positive harmonic functions, and the fact that $u_{2}$ increases in time,

$$
u_{2}(y, s) \leq c(n) C_{2} \gamma^{2} \epsilon \text { in } B_{\gamma \epsilon}\left(z_{0}\right) \times\left[0, \tau_{0}\right]
$$

where $c(n)$ is the dimensional constant from the harnack inequality.

Due to Lemma $3.1 \Gamma\left(u_{2}\right)$ is in $M \epsilon$-neighborhood of $l_{q, r}$. Hence at time $\tau_{1}=\tau_{0}-(M+\gamma) \epsilon$, the ball $B_{\gamma \epsilon}\left(z_{0}\right)$ is in the zero set of $u_{2}$. Now a barrier argument as in previous step using (3.17) in the domain $B_{\gamma \epsilon}\left(z_{0}\right) \times\left[\tau_{1}, \tau_{0}\right]$ yields that if $C_{2}$ is small enough then $B_{\gamma \epsilon / 2}\left(z_{0}\right)$ is in the zero set of $u_{2}\left(\cdot, \tau_{0}\right)$, contradicting (3.16).

3. Now we proceed to prove (3.13). Due to Lemma 3.1, $u_{2}\left(x, t+\gamma^{2} \epsilon\right) \leq P_{q, r}\left(x, t+\left(M+\gamma^{2}\right) r^{-1} \epsilon\right) \leq P_{q, r}(x, t)+2 M \epsilon \leq u_{2}(x, t)+2 M \epsilon$. 
It follows from (3.11) that

$$
\left(\tilde{u}_{2}-\tilde{u}_{1}\right)(x, t) \geq-2 M \epsilon \text { in } \mathcal{S} .
$$

Also observe that, due to the boundary condition of $u_{1}$ and $u_{2}$,

$$
\tilde{u}_{2}(x, t)-\tilde{u}_{1}(x, t) \geq 0 \text { for } x \cdot q \leq-2 M \epsilon_{0} / \gamma, \quad 0 \leq t \leq 2 M \epsilon_{0} / \gamma .
$$

Thus for $t \in\left[t_{0}-3 M \epsilon, t_{0}\right]$,

$$
v(\cdot, t):=\inf _{B_{\gamma \epsilon}(\cdot)} u_{2}(\cdot, t)-\tilde{u}_{1}(\cdot, t)
$$

is a superharmonic function in $\Omega_{t}\left(\tilde{u}_{1}\right) \cap \mathcal{S}$, with boundary data bigger than $C_{2} \gamma^{2} \epsilon$ on $\Gamma_{t}\left(\tilde{u}_{1}\right) \cap \mathcal{S}$, bigger than zero on the strip $\left\{x: x \cdot q=-2 M \epsilon_{0} / \gamma\right\}$ and bigger than $-M \epsilon$ on $\partial \mathcal{S}$.

Hence $v(x, t) \geq h(x)$ in $\Omega_{t}\left(\tilde{u}_{1}\right) \times\left[t_{0}-3 M \epsilon, t_{0}\right]$, where $h$ is a harmonic function in

$$
\Sigma:=\left\{-2 M \epsilon_{0} / \gamma \leq x \cdot q \leq M \epsilon+x_{0} \cdot q\right\} \cap \mathcal{S}
$$

with boundary data $\gamma^{2} \epsilon$ on the upper strip, zero on the bottom strip, and $-M \epsilon$ on the lateral boundary. Since the width of $S$ is $N \epsilon$ with $N>1 / \gamma^{2}$, it follows from a straightforward computation that if $\gamma$ is sufficiently small then $h \geq 0$ on $B_{3 M \epsilon}\left(x_{0}\right)$.

5. We proceed with the proof of the proposition. By previous argument we have

$$
\tilde{u}_{1}(x, t) \leq \inf _{B_{\gamma \epsilon}} u_{2}(x, t) .
$$

in $B_{3 M \epsilon}\left(x_{0}\right) \times\left[t_{0}-3 M \epsilon, t_{0}\right]$. Let $x_{1}:=x_{0}+(M+2) \epsilon q$, and let $R=$ $B_{2 M \epsilon}\left(x_{1}\right)-B_{\epsilon}\left(x_{1}\right)$.

Define

$$
w(x, t):=\inf _{y \in B_{\gamma \epsilon \varphi(x)}(x)} u_{2}(y, t)
$$

where $\varphi$ defined in $R$ satisfies the following properties:

$$
\begin{cases}(a) \quad \Delta\left(\varphi^{-A_{n}}\right)=0 & \text { in } R ; \\ (b) \quad \varphi=B_{n} & \text { on } \partial B_{\epsilon}\left(x_{1}\right) ; \\ (c) \quad \varphi=1 & \text { in } \partial B_{2 M \epsilon}\left(x_{1}\right) .\end{cases}
$$


Fix $A_{n}>0$, a sufficiently large dimensional constant. Then Lemma 9 in [C1] yields that $w$ is superharmonic in $\Omega_{t}(w) \cap R$ for $0 \leq t \leq 2 M \epsilon_{0} / \gamma$. Choose $B_{n}$ (depending on $A_{n}$ ) sufficiently large that $\varphi\left(x_{0}\right)>C_{1}$. Note that $|D \varphi| \leq C_{4}$ where $C_{4}$ depends on $A_{n}, B_{n}$ and $M$.

6. Now we compare $w$ and $\tilde{u}_{1}$ in $\diamond:=R \times\left[-2 M \epsilon+t_{0}, t_{0}\right]$. Due to (3.18) and the fact that $\tilde{u} \leq \tilde{u_{2}}=0$ in $B_{\epsilon}\left(x_{1}\right), \tilde{u}_{1} \leq w$ on $\partial R \times\left[-2 M \epsilon+t_{0}, t_{0}\right]$. Moreover at $t=-2 M \epsilon+t_{0}$ the positive phase of $\tilde{u}_{1}$ is outside of $R$, and thus $\tilde{u} \equiv 0 \leq w$ in $R$. Hence $\tilde{u}_{1} \leq w$ on the parabolic boundary of $\diamond$. However, since $\varphi\left(x_{0}\right)>C_{1}, \tilde{u}_{1}$ crosses $w$ from below in $\diamond$. This will be a contradiction to Theorem 1.7 if we show that $w$ is a supersolution of $(P)_{\epsilon}$ in $\diamond$.

7. Since $w$ is superharmonic in its positive phase, to prove that $w$ is a supersolution we only have to check the free boundary condition. Suppose there is a $C^{2,1}$ function $\psi$ such that $w-\psi$ has a local minimum at $\left(x_{1}, t_{1}\right) \in$ $\Gamma(w) \cap \diamond$ in $\bar{\Omega}(w) \cap \Sigma$ with $|D \psi|\left(x_{1}, t_{1}\right) \neq 0$. By definition, there is $y_{1} \in \Gamma_{t}\left(u_{2}\right)$ such that

$$
u_{2}\left(y_{1}, t_{1}\right)=w\left(x_{1}, t_{1}\right)
$$

and set

$$
u_{3}(x, t):=u_{2}(x+\nu \gamma \epsilon \varphi(x), t) \quad \nu=\frac{y_{1}-x_{1}}{\left|y_{1}-x_{1}\right|} .
$$

Then $u_{3}-\psi$ has a local minimum at $\left(x_{1}, t_{1}\right)$ in $\bar{\Omega}\left(u_{3}\right) \cap \diamond$. Formally speaking, on its free boundary $u_{3}(x, t)$ satisfies

$$
\begin{aligned}
\left(\left(u_{3}\right)_{t}-g(x / \epsilon)\left|D u_{3}\right|^{2}\right) & \left(x_{1}, t_{1}\right) \\
& \geq\left(u_{2}\right)_{t}\left(y_{1}, t_{1}\right) \\
& -(1+C \gamma|\varphi|+C \gamma \epsilon|D \varphi|)\left(x_{1}\right) g\left(\frac{y_{1}}{\epsilon}\right)\left|D u_{2}\right|^{2}\left(y_{1}, t_{1}\right) \\
& \geq\left(\left(u_{2}\right)_{t}-(1+C \gamma) g\left(\frac{y_{1}}{\epsilon}\right)\left|D u_{2}\right|^{2}\right)\left(y_{1}, t_{1}\right) \\
& \geq 0
\end{aligned}
$$

if $C(n)$ in (3.7) is large enough. Therefore we obtain $\psi_{t}-g(\cdot / \epsilon)|D \psi|^{2} \geq 0$ at $\left(x_{1}, t_{1}\right)$ and $w$ is a supersolution of $(P)_{\epsilon}$ in $\diamond$. For rigorous argument proving that $w$ is a supersolution of $(P)_{\epsilon}$ in $\diamond$, one can argue as in the proof of Lemma 3.4 in [K2].

Remark Note that the condition $\epsilon<\epsilon_{0}$ is used to guarantee that the domain $\mathcal{O}$, with which $u_{1}$ is defined, remains a subset of $Q_{1}$.

Parallel arguments yield the corresponding result for $\underline{u}_{\epsilon ; q, r}$ : 
Proposition 3.6. Suppose $q, N$ as given in Proposition 3.4. Suppose $r=$ $r_{0}-C(n) \gamma>r_{0} / 2$, where $r_{0}:=\underline{r}(q)$ and $C(n)>0$ is a sufficiently large dimensional constant. If $1 / N<\gamma^{2}$ then for $\epsilon<\epsilon_{0}=\frac{\gamma^{2}}{8 n M N}$

$$
d\left(\Gamma_{t}\left(\underline{u}_{\epsilon ; q, r}\right), l_{q, r}(t) \cap B_{1 / 4}(0)\right)>M \epsilon_{0}
$$

for $\frac{M \epsilon_{0}}{\gamma} \leq t \leq 1$, where $M$ is the dimensional constant given in Lemma 2.9.

We are now ready to prove that $\bar{r}(q)=\underline{r}(q)$ for any unit vector $q \in \mathbb{R}^{n}$. First we will show that $\bar{r}(q) \leq \underline{r}(q)$. The following elementary lemma is given as Exercise 1.15-1.16 of [A].

Lemma 3.7. (a) Given a real $x$ and an integer $N>1$, there exits integers $h$ and $k$ with $0<k \leq N$ such that $|k x-h|<1 / N$.

(b) If $x$ is irrational there are infinitely many rational numbers $h / k$ with $k>0$ such that $|x-h / k| \leq 1 / k^{2}$.

Next we consider general unit vector $q=m\left(e_{1}+\alpha_{2} e_{2}+\ldots \alpha_{n} e_{n}\right) \in \mathbb{R}^{n}$, $1 / n \leq|m| \leq 1,\left|\alpha_{k}\right| \leq 1, k=2, \ldots, n$.

Lemma 3.8. $\bar{r}(q) \leq \underline{r}(q)$ for any unit vector $q \in \mathbb{R}^{n}$.

Proof. 1. Note that the lemma is shown for rational vectors in Lemma 3.2.

2 . We will only prove $\bar{r}(q) \leq \underline{r}(q)$ for the case where the coefficients $\alpha_{i}$ are all irrational, other cases can be proven similar.

3. Take any $\gamma>0$, where $C(n)$ is as given in Proposition 3.4. Due to Lemma 3.7 there are integers $a_{2}, N_{2}$ prime to each other such that

$$
\left|\alpha_{1}-a_{2} / N_{2}\right| \leq \gamma^{5} / N_{2}, \quad N_{2}>\gamma^{-2} .
$$

Again due to Lemma 3.7 for any $a>0$, there exist $a_{i}, N_{i} \in \mathbb{Z}, 1 \leq N_{i} \leq$ $\frac{N_{2}}{2 a}$ such that

$$
\left|\alpha_{i}-a_{i} / N_{i}\right| \leq \frac{a}{N_{2} N_{i}}, i=3, \ldots, n .
$$

choose $a>0$ small enough so that $N_{i}>\gamma^{-3}$ for $i=3, \ldots, n$. Let $N=\max N_{i}, i=2, \ldots, n$. Then

$$
\left|\alpha_{i}-a_{i} / N_{i}\right| \leq \frac{\gamma^{3}}{2 N}, \quad i=2, \ldots, n .
$$


Now choose

$$
\tilde{q}=\tilde{q}(\gamma):=m\left(e_{1}+\frac{a_{2}}{N_{2}} e_{2}+\ldots+\frac{a_{n}}{N_{n}} e_{n}\right)
$$

and compare $\bar{u}_{\epsilon ; q, r}$ with $\bar{u}_{\epsilon ; \tilde{q}, r}$ at $r=\bar{r}(\tilde{q})+C(n) \gamma$, where $C(n)$ is given as in Proposition 3.4. Due to Proposition 3.4 applied to $\tilde{q}$ we obtain that, for $\epsilon \leq \epsilon_{0}, \epsilon_{0}=\frac{\gamma^{2}}{8 n M N}$,

$$
d\left(\Gamma_{1}\left(\bar{u}_{\epsilon ; \mu, r}\right), l_{\mu, r}(1) \cap B_{1 / 2}(0)\right)>\frac{M}{2} \epsilon_{0},
$$

and thus $\bar{r}(\mu) \leq \bar{r}(\tilde{q})+C(n) \gamma$ for any unit vector $\mu$ such that $|\mu-\tilde{q}| \leq \frac{\gamma^{2}}{16 n N}$, including $q$ due to (3.19).

Similarly Proposition 3.6 yields that at $r=\underline{r}(\tilde{q})-C(n) \gamma$ where $C(n)$ is given as in Proposition 3.6,

$$
\left.d\left(\Gamma_{1}\left(\underline{u}_{\epsilon ; \mu, r}\right), l_{q, r}(1)\right) \cap B_{1 / 2}(0)\right)>\frac{M}{2} \epsilon_{0}
$$

and thus $\underline{r}(\mu) \geq \underline{r}(\tilde{q})-C(n) \gamma$ for any unit vector $\mu$ such that $|\mu-\tilde{q}| \leq$ $\frac{\gamma^{2}}{16 n N}$, including $q$ due to $(3.19)$.

Hence it follows that

$$
\bar{r}(q) \leq \liminf _{\gamma \rightarrow 0} \bar{r}(\tilde{q}) \leq \limsup _{\gamma \rightarrow 0} \underline{r}(\tilde{q}) \leq \underline{r}(q) .
$$

Let $C(n)$ be the maximum of dimensional constants given in Propositions 3.4 and 3.6.

Corollary 3.9. For any unit vector $q \in \mathbb{R}^{n}$ and for small $\gamma>0$, there exists $0<\epsilon_{0}=\epsilon(q, \gamma)<\frac{\gamma^{4}}{n M}$ such that if $r=\bar{r}(q)+C(n) \gamma(r=\underline{r}(q)-C(n) \gamma)$ then for $0<\epsilon \leq \epsilon_{0}$

$$
d\left(y, l_{q, r}(t)\right)>\frac{M \epsilon_{0}}{2} \text { for any } y \in \Gamma_{t}\left(\bar{u}_{\epsilon ; q, r}\right) \cap B_{1 / 4}(0)
$$

( for any $y \in \Gamma\left(\underline{u}_{\epsilon ; q, r}\right) \cap B_{1 / 4}(0)$ )

for $\frac{M \epsilon_{0}}{\gamma} \leq t \leq 1$, where $M$ is given as in Lemma 2.9.

Lemma 3.10. $\underline{r}(q) \leq \bar{r}(q)$ for any unit vector $q \in \mathbb{R}^{n}$. 
Proof. Suppose not. Then for some $\gamma>0, \underline{r}(q)=\bar{r}(q)+2 C(n) \gamma$. We compare

$$
u_{1}(x, t)=\bar{u}_{\epsilon ; q, r}(x, t) \text { and } u_{2}(x, t)=\underline{u}_{\epsilon ; q, r}\left(x, t-4 \epsilon_{0}\right)
$$

at $r=\bar{r}(q)+C(n) \gamma$ in $\mathcal{D}(q) \times[0,1]$, where $\epsilon_{0}=\epsilon_{0}(q, \gamma)$ is given as in Corollary 3.9. By Corollary 3.9, $u_{2}$ crosses $u_{1}$ from below in $Q_{1}$ at $\left(x_{0}, t_{0}\right)$, $t_{0} \in\left[4 \epsilon_{0}, M \epsilon_{0} / \gamma\right]$. By Lemma 2.4 and the boundary data of $u_{1}$ and $u_{2}$ on the parabolic boundary of $Q_{1}, x_{0}$ is more than $2 \epsilon_{0}$-away from the lateral boundary of $\bar{D}(q)$ and on $\Gamma\left(u_{1}\right) \cap \Gamma\left(u_{2}\right)$.

Observe that, by definition of $\bar{u}_{\epsilon ; q, r}$, for any vector $\mu \in a \epsilon_{0} \mathbb{Z}^{n}, 0<a<1$ satisfying $\mu \cdot q \geq 0$ and $|\mu| \leq 1-a$

$$
a \bar{u}_{\epsilon_{0} ; q, r}\left(\frac{x-\mu}{a}, \frac{t-r \mu \cdot q}{a}\right) \geq \bar{u}_{a \epsilon_{0} ; q, r}(x, t) \text { in } a Q_{1}+(\mu, r \mu \cdot q) .
$$

Therefore by Corollary 3.9 if $\epsilon<\epsilon_{0}^{2}$ then $\Gamma\left(u_{1}\right)$ and $\Gamma\left(u_{2}\right)$ is away from $l_{q, r}$ in $\diamond=\left(1-\epsilon_{0}\right) \mathcal{D}(q) \times\left[\epsilon_{0}, 1\right]$, and therefore $u_{1}$ and $u_{2}$ are a solutions of $(P)_{\epsilon}$ in $\diamond$. This contradicts Theorem 1.7.

Corollary 3.11. $\bar{r}(q)=\underline{r}(q)$ for any unit vector $q \in \mathbb{R}^{n}$.

For a unit vector $q \in \mathbb{R}^{n}$, we define

$$
r(q):=\bar{r}(q)=\underline{r}(q) .
$$

Lemma 3.12. $r(q)$ is continuous.

Proof. Due to Corollary 3.9 it follows that if $\left|q_{1}-q_{2}\right| \leq \epsilon_{0}\left(q_{1}, \gamma\right)$ where $\epsilon_{0}\left(q_{1}, \gamma\right)$ is as given in Corollary 3.9 then $\left|r\left(q_{1}\right)-r\left(q_{2}\right)\right| \leq C(n) \gamma$, which yields our conclusion.

\section{Convergence to the limiting problem}

Consider the free boundary problem

$$
\begin{cases}-\Delta u=0 & \text { in }\{u>0\} \cap Q, \\ u_{t}-r(\nu)|\nabla u|^{2}=0 & \text { on } \partial\{u>0\} \cap Q\end{cases}
$$

with initial data $u_{0}$ and fixed boundary data on $\partial K$, where $Q, u_{0}$ and $\nu$ is as given in $(P)_{\epsilon}$ in the introduction and $r(q)$ is the continuous function on 
$\left\{q \in \mathbb{R}^{n}:|q|=1\right\}$ defined in (3.20). Note that the existence and uniqueness results in section 1 , in particular Theorem 1.7 applies to $(P)$.

We assume that $\Omega_{0}$ satisfies

$$
\operatorname{Int}\left(\bar{\Omega}_{0}\right)=\Omega_{0}, \quad\left|D u_{0}\right| \neq 0 \text { on } \Gamma_{0},
$$

so that Theorem 1.8 (a) applies and there exists a unique viscosity solution of $(P)$.

Consider solutions $\left\{u^{\epsilon}\right\}$ of free boundary problem $(P)_{\epsilon}$ with initial data $u_{0}$ and fixed boundary data 1 . Let us define

$$
u_{1}(x, t):=\left(\lim _{\epsilon_{0}, r \rightarrow 0} \sup \left\{u^{\epsilon}(y, s): \epsilon<\epsilon_{0}, s \geq 0,|(x, t)-(y, s)| \leq r\right\}\right)^{*}
$$

and

$$
u_{2}(x, t)=\left(\lim _{\epsilon_{0}, r \rightarrow 0} \inf \left\{u^{\epsilon}(y, s): \epsilon<\epsilon_{0}, s \geq 0,|(x, t)-(y, s)| \leq r\right\}\right)_{*} .
$$

Note that $u_{1}(x, 0)=u_{2}(x, 0)=u_{0}(x)$, since $\Omega_{0}(u)=\cap_{t>0} \Omega_{t}(u)$ at $t=0$ due to the condition (4.1). Our goal in this section is to prove that $u_{1}$ and $u_{2}$ are respectively sub- and supersolutions of $(P)$.

Lemma 4.1. Suppose $\left(x_{0}, t_{0}\right) \in \Gamma\left(u^{\epsilon}\right)$. Then there exists $c=c(n)>0$ and $r_{0}=r_{0}(d)>0$, where $d=d\left(x_{0}, \Omega\left(u_{0}\right)\right)$ such that if $r<\frac{r_{0}}{2}$ then

$$
s\left(r, \epsilon ; x_{0}, t_{0}\right):=\sup _{B_{2 r}\left(x_{0}\right)} u^{\epsilon}\left(\cdot, t_{0}\right) \geq c \frac{r^{2}}{t_{0}} .
$$

Proof. Consider a point $x_{0} \in \Gamma_{t_{0}}\left(u_{\epsilon}\right)$ with $d\left(x, \Omega_{0}(u)\right)>2 r$. Take $\tau>0$ such that $u_{\epsilon}(\cdot, t) \equiv 0$ in $B_{2 r}\left(x_{0}\right)$. Since $u^{\epsilon}$ is increasing in time, one can show by the barrier argument in $B_{2 r}\left(x_{0}\right) \times\left[\tau, t_{0}\right]$ that if

$$
s\left(r, \epsilon, x_{0}, t_{0}\right) \leq c \frac{r^{2}}{t_{0}}
$$

where $c$ is a sufficiently small dimensional constant, then $\Gamma\left(u_{\epsilon}\right)$ will not reach $x_{0}$ by the time $t=t_{0}$, yielding a contradiction.

Theorem 4.2. $u_{1}$ and $u_{2}$ are respectively a subsolution and a supersolution of $(P)$ with initial data $u_{0}$ and fixed boundary data 1 . In particular $u:=$ $u_{1}=u_{2}$ and $\left\{u_{\epsilon}\right\}$ uniformly converges to $u$ as $\epsilon \rightarrow 0$, where $u$ is the unique viscosity solution of $(P)$ with initial data $u_{0}$ and fixed boundary data 1. 


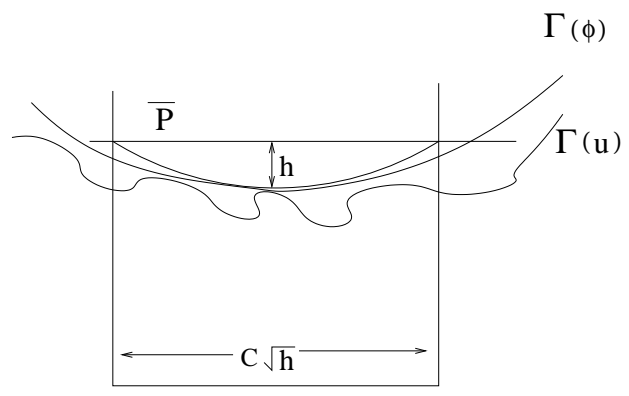

$\mathrm{P}$

Figure 6 .

Proof. The second assertion follows from the first assertion and Theorem 1.7 for $(P)$.

To prove the first assertion, suppose $\phi$ touches $u_{1}$ from above at $P_{0}=$ $\left(x_{0}, t_{0}\right) \in \Gamma\left(u_{1}\right)$ with $|D \phi|\left(P_{0}\right) \neq 0$ and

$$
\max \left[-\Delta \phi, \phi_{t}-r(\nu)|D \phi|^{2}\right]\left(P_{0}\right)=C(n) \gamma|D \phi|^{2}\left(P_{0}\right)>0,
$$

for some $\gamma>0$, where $\nu=\frac{q}{|q|}, q=-D \phi\left(x_{0}, t_{0}\right)$. Without loss of generality we may assume that $|q|=1$ - otherwise a scaling argument applies, and that the maximum is zero and strict- otherwise consider, with small $\delta>0$,

$$
\tilde{\phi}(x, t):=\phi(x, t)-\phi\left(x_{0}, t_{0}\right)+\delta\left(x-x_{0}\right)^{4}+\delta\left(t-t_{0}\right)^{2} .
$$

Let

$$
P_{h}(x, t):=\left(r\left(t-t_{0}\right)-\nu \cdot\left(x-x_{0}-h \nu\right)\right)_{+}, r=\frac{\phi_{t}}{|D \phi|}\left(x_{0}, t_{0}\right) .
$$

Since $\phi$ is smooth with $|D \phi|\left(P_{0}\right) \neq 0, \Gamma(\phi)$ has an exterior ball at $P_{0}$ and thus for sufficiently small $h>0$

$$
u_{1}(x, t) \leq \phi(x, t) \prec P_{h} \text { in } B_{C h^{1 / 2}}\left(x_{0}\right) \times\left[t_{0}-C h^{1 / 2}, t_{0}\right]
$$

(see Figure 6.)

Choose $h \leq C^{2} \epsilon_{0}^{2}(q, \gamma)$ where $C$ is given as in (4.2) and $\epsilon_{0}(q, \gamma)$ is given as in Corollary 3.9. By definition of $u_{1}$ and by Lemma 4.1, there exists a sequence $\epsilon_{k} \rightarrow 0$ such that $u_{\epsilon_{k}} \prec P_{h}$ in $C h^{1 / 2}$-neighborhood of $\left(x_{0}, t_{0}\right)$ and

$$
d\left(\left(x_{0}, t_{0}\right), \Gamma\left(u_{\epsilon_{k}}\right)\right) \rightarrow 0 \text { as } k \rightarrow \infty,
$$

However after a scaling argument, Corollary 3.9 yields that $\Gamma\left(u_{\epsilon_{k}}\right)$, for sufficiently small $\epsilon_{k}$, should stay away from $\Gamma\left(P_{h}\right)$ by $C \epsilon_{0} h^{1 / 2}$ in $C / 2 h^{1 / 2}$ neighborhood of $\left(x_{0}, t_{0}\right)$, which is a contradiction since

$$
d\left(x_{0}, \Gamma\left(P_{h}\right)\right)=h<C \epsilon_{0} h^{1 / 2} .
$$




\section{Homogenization of Stefan-type problems}

In this section we will consider the limiting behavior of $\left\{u_{\epsilon}\right\}$ solving the Stefan-type problem

$$
\left\{\begin{array}{lll}
u_{t}^{\epsilon}-\Delta u^{\epsilon}=0 & \text { in } \quad & \left\{u^{\epsilon}>0\right\}, \\
u_{t}-g(x / \epsilon)\left|D u^{\epsilon}\right|^{2}=0 & \text { on } & \partial\left\{u^{\epsilon}>0\right\},
\end{array}\right.
$$

in $\mathbb{R}^{n} \times[0, \infty)$ with initial data $u_{0}(x)$. Here and $g$ is as given in $(P)_{\epsilon}$. To ensure that $u^{\epsilon}$ is behaves smoothly near $t=0$ we assume that $u_{0}$ satisfies

$$
u_{0} \in C^{2}\left(\overline{\left\{u_{0}>0\right\}}\right) \text { and }\left|D u_{0}\right|, \Delta u_{0}>C_{0} \text { near } \Gamma_{0}:=\partial\left\{u_{0}>0\right\} .
$$

As before, for uniqueness and existence results we consider the generalized problem

$$
\begin{cases}u_{t}^{\epsilon}-\Delta u^{\epsilon}=0 & \text { in } \quad\left\{u^{\epsilon}>0\right\} \\ u_{t}^{\epsilon}-g(x / \epsilon, \nu)\left|D u^{\epsilon}\right|^{2}=0 & \text { on } \quad \partial\left\{u^{\epsilon}>0\right\}\end{cases}
$$

where $g, \nu$ is as given in $(\tilde{P})_{\epsilon}$. Here we extend the notion of viscosity solutions for the Stefan problem $(g \equiv 1)$ in section 4 of $[\mathrm{K} 1]$ to define the viscosity solutions of $(\tilde{P} 2)_{\epsilon}$. Let $\Sigma$ be an open set in $\mathbb{R}^{n} \times[0, \infty)$.

Definition 5.1. A nonnegative upper semicontinuous function $u$ in $\bar{\Sigma}$ is a viscosity subsolution of $(\tilde{P} 2)_{\epsilon}$ in $\Sigma$ if for every $\phi \in C^{2,1}(\Sigma)$ such that $u-\phi$ has a local maximum in $\overline{\Omega(u)} \cap\left\{t \leq t_{0}\right\} \cap \Sigma$ at $\left(x_{0}, t_{0}\right)$ then

(i) $\quad\left(\phi_{t}-\Delta \phi\right)\left(x_{0}, t_{0}\right) \leq 0 \quad$ if $u\left(x_{0}, t_{0}\right)>0$.

(ii) if $\left(x_{0}, t_{0}\right) \in \Gamma(u),|\nabla \phi|\left(x_{0}, t_{0}\right) \neq 0$ and $\left(\phi_{t}-\Delta \varphi\right)\left(x_{0}, t_{0}\right)>0$,

then

$$
\left(\phi_{t}-g\left(x_{0} / \epsilon, \nu\right)|D \phi|^{2}\right)\left(x_{0}, t_{0}\right) \leq 0,
$$

where $\nu=-\frac{D \phi}{|D \phi|}\left(x_{0}, t_{0}\right)$. 
Definition 5.2. A nonnegative lower semicontinuous function $u$ in $\bar{\Sigma}$ is a viscosity supersolution of $(\tilde{P} 2)_{\epsilon}$ in $\Sigma$ if for every $\phi \in C^{2,1}(\Sigma)$ such that $u-\phi$ has a local maximum in $\overline{\Omega(u)} \cap\left\{t \leq t_{0}\right\} \cap Q$ at $\left(x_{0}, t_{0}\right)$ then

(i) $\left(\phi_{t}-\Delta \phi\right)\left(x_{0}, t_{0}\right) \leq 0 \quad$ if $u\left(x_{0}, t_{0}\right)>0$.

(ii) if $\left(x_{0}, t_{0}\right) \in \Gamma(u),|\nabla \phi|\left(x_{0}, t_{0}\right) \neq 0$ and $\left(\phi_{t}-\Delta \phi\right)\left(x_{0}, t_{0}\right)>0$,

then

$$
\left(\phi_{t}-g\left(x_{0} / \epsilon, \nu\right)|D \phi|^{2}\right)\left(x_{0}, t_{0}\right) \leq 0,
$$

where $\nu=-\frac{D \phi}{|D \phi|}\left(x_{0}, t_{0}\right)$.

Definition 5.3. (a) $u$ is a viscosity subsolution of $(\tilde{P} 2)_{\epsilon}$ with initial data $u_{0}$ if $u$ is a viscosity subsolution in $\mathbb{R}^{n} \times[0, \infty)$ with $u(x, 0)=u_{0}(x)$ and $\overline{\Omega(u)} \cap\{t=0\}=\overline{\Omega\left(u_{0}\right)}$.

(b) $u$ is a viscosity supersolution of $(\tilde{P} 2)_{\epsilon}$ with initial data $u_{0}$ if $u$ is a viscosity supersolution in $\mathbb{R}^{n} \times[0, \infty)$ with $u=u_{0}$ at $t=0$.

A parallel argument as in section 4 of [K1] yields the following theorem on the solutions of $(\tilde{P} 2)_{\epsilon}$ :

Theorem 5.4. Theorem 1.7 holds between a subsolution and a supersolution of $(\tilde{P} 2)_{\epsilon}$. Furthermore there exists a unique viscosity solution of $(\tilde{P} 2)_{\epsilon}$ in $\mathbb{R}^{n} \times[0, \infty)$ with initial data $u_{0}$ satisfying (5.1).

Consider

$$
\left\{\begin{array}{llc}
u_{t}-\Delta u=0 & \text { in } & \{u>0\} \\
u_{t}-r(\nu)|D u|^{2}=0 & \text { on } & \partial\{u>0\},
\end{array}\right.
$$

where $r(\nu)$ is as defined in (3.20). Let $u$ be the unique viscosity solution of $(P 2)$ with initial data $u_{0}$. Below we show that $u^{\epsilon}$ solving $(P 2)_{\epsilon}$ uniformly converges to $u$ as $\epsilon \rightarrow 0$. First we prove a nondegeneracy property of $\left\{u^{\epsilon}\right\}$.

Lemma 5.5. Suppose $\left(x_{0}, t_{0}\right) \in \Gamma\left(u^{\epsilon}\right), t_{0}>0$. Then for any $\epsilon>0, d_{x_{0}, t_{0}}:=$ $d\left(x_{0}, \Omega_{0}\right) \geq c\left(t_{0}\right)>0$ and for any $0<r<d_{x_{0}, t_{0}}$,

$$
\sup _{B_{r}\left(x_{0}\right) \times\left[0, t_{0}\right]} u^{\epsilon}(y, s) \geq \frac{c_{0} r^{2}}{t_{0}} .
$$

where $c_{0}=c_{0}\left(t_{0}, n\right)$. 
Proof. 1.The first assertion follows from (5.1). In fact due to (5.1), for $C_{0}$ given in (5.1) and for sufficiently small $t_{0}$ we have

$$
d_{x_{0}, t_{0}} \geq d\left(\Gamma_{t_{0}}\left(u^{\epsilon}\right), \Omega_{0}\right)>\frac{C_{0}}{2} t_{0}>0 .
$$

2. Let us choose $C\left(t_{0}\right)>0$ such that

$$
u_{0}(x) \prec\left(1+C\left(t_{0}\right)\right) u^{\epsilon}\left(x, t_{0}\right) .
$$

(Such $C\left(t_{0}\right)$ exists since the positive phase of $u^{\epsilon}$ expands in time.)

If (5.2) is not true, then due to (5.3)

$$
u^{\epsilon} \leq \frac{c_{0}\left(1+C\left(t_{0}\right)\right) r^{2}}{t_{0}} \text { in } B_{r}\left(x_{0}\right) \times\left[0, t_{0}\right]
$$

for some $r \in\left(0, d_{x_{0}, t_{0}}\right)$. Since $u^{\epsilon}(\cdot, 0) \equiv 0$ in $B_{r}\left(x_{0}\right)$, a barrier argument with a radially symmetric barrier function in $B_{r}\left(x_{0}\right) \times\left[0, t_{0}\right]$ using (5.4) yields that if $c_{0} \leq c(n)\left(1+C\left(t_{0}\right)\right)^{-1}$ with sufficiently small $c(n)$ then $\Gamma\left(u^{\epsilon}\right)$ does not reach $x_{0}$ by $t=t_{0}$, a contradiction.

Let us define

$u_{1}(x, t):=\left(\lim _{\epsilon_{0}, r \rightarrow 0} \sup \left\{u^{\epsilon}(y, s): \epsilon<\epsilon_{0}, s \geq 0,(y, s) \in B_{r}(x) \times[t-r, t+r]\right\}\right)^{*}$

and

$u_{2}(x, t):=\left(\lim _{\epsilon_{0}, r \rightarrow 0} \inf \left\{u^{\epsilon}(y, s): \epsilon<\epsilon_{0}, s \geq 0,(y, s) \in B_{r}(x) \times[t-r, t+r]\right\}\right)_{*}$.

Theorem 5.6. $u_{1}$ and $u_{2}$ are respectively a viscosity sub- and supersolution of $(P 2)$ with initial data $u_{0}$. In particular if $u$ is the unique viscosity solution of (P2) with initial data $u_{0}$, then $u_{1}=u_{2}$ and $\left\{u^{\epsilon}\right\}$ uniformly converges to $u$ as $\epsilon \rightarrow 0$.

Proof. 1. The second assertion follows from the first. It is easy to check that $u_{1}=u_{2}=u_{0}$ at $t=0$, due to (5.1).

2. Suppose $u_{1}-\phi$ has a local maximum zero in $\bar{\Omega}\left(u_{1}\right)$ at $\left(x_{0}, t_{0}\right) \in \Gamma\left(u_{1}\right)$ with $|D \phi|\left(x_{0}, t_{0}\right) \neq 0$ and

$$
\min \left(\phi_{t}-\Delta \phi, \phi_{t}-r(\nu)|D \phi|^{2}\right)\left(x_{0}, t_{0}\right)>0,
$$

where $\nu=\frac{q}{|q|}$ and $q=-D \phi\left(x_{0}, t_{0}\right)$, in a local neighborhood $B_{k}\left(x_{0}\right) \times\left[t_{0}-\right.$ $\left.k, t_{0}\right]$. As in the proof of Theorem 4.2 , without loss of generality we may assume that $|q|=1$ and the maximum is strict. Due to Lemma 5.5,

$$
\bar{\Omega}\left(u_{1}\right)=\limsup _{\epsilon \rightarrow 0} \bar{\Omega}\left(u^{\epsilon}\right)
$$


and thus there is a sequence $\epsilon_{i} \rightarrow 0$ such that $u^{\epsilon_{i}}-\phi$ has maximum in $B_{k}\left(x_{0}\right) \times\left[t_{0}-k, t_{0}\right]$ at $\left(x_{i}, t_{i}\right) \in \Gamma\left(u^{\epsilon_{i}}\right)$ with $\left(x_{i}, t_{i}\right) \rightarrow\left(x_{0}, t_{0}\right)$ as $i \rightarrow \infty$. Since $\phi$ is smooth with $|D \phi|\left(x_{0}, t_{0}\right) \neq 0$, there exists $C_{0}>0$ such that for $0<\epsilon_{i}<<h<<1$ and for any vector $\xi \in \mathbb{R}^{n}$ with $|\xi| \leq 2 \epsilon_{i}$,

$$
u^{\epsilon_{i}}(x, t) \prec P_{q, r}\left(x-x_{0}-C_{0} h \nu+\xi, t-t_{0}\right) \text { in } B_{h^{1 / 2}}\left(x_{0}\right) \times\left[t_{0}-h^{1 / 2}, t_{0}\right]
$$

where $r=\phi_{t}\left(x_{0}, t_{0}\right)$.

Consider

$$
w_{\epsilon}(x, t):=\bar{u}_{h^{-1 / 2} \epsilon ; q, r}\left(\frac{x-x_{0}+r h^{1 / 2} \nu-C_{0} h \nu+\xi_{\epsilon}}{h^{1 / 2}}, \frac{t-t_{0}+h^{1 / 2}}{h^{1 / 2}}\right),
$$

where $\bar{u}_{h^{1 / 2} \epsilon ; q, r}$ is as in Definition 2.1 and $\xi_{\epsilon} \in \mathbb{R}^{n}$ is chosen such that $x_{0}-r h^{1 / 2} \nu-\xi_{\epsilon} \in h^{1 / 2} \epsilon \mathbb{Z}^{n}$ and $\left|\xi_{\epsilon}\right| \leq 2 \epsilon$. Note that $w_{\epsilon}$ solves $(P)_{\epsilon}$ in $Q_{1}$ away from the contact set

$$
\Gamma\left(w_{\epsilon}\right) \cap\left(l_{q, r}\left(t-t_{0}\right)+x_{0}-\xi_{\epsilon}\right)
$$

and $w_{\epsilon}$ is harmonic in its positive phase, and thus

$$
\left(w_{\epsilon}\right)_{t}-\Delta w_{\epsilon}=\left(w_{\epsilon}\right)_{t} \geq 0 \text { in } \Omega\left(w_{\epsilon}\right) .
$$

Therefore $w_{\epsilon}$ is a supersolution of $(P 2)_{\epsilon}$ away from $l_{q, r}\left(x-x_{0}-C_{0} h \nu+\right.$ $\left.\xi_{\epsilon}, t-t_{0}\right)$. Due to $(5.5)$, we have

$$
u^{\epsilon_{i}} \leq w_{\epsilon_{i}} \text { in } B_{h^{1 / 2}}\left(x_{0}\right) \times\left[t_{0}-h^{1 / 2}, t_{0}\right] .
$$

On the other hand, since $r=r(\nu)+C(n) \gamma$ for some $\gamma>0$ where $C(n)$ is as given in Corollary 3.9, Corollary 3.9 applies to $\bar{u}_{h^{-1 / 2} \epsilon ; q, r}$ to yield that

$$
d\left(\Gamma_{t_{0}}\left(w_{\epsilon}\right) \cap \frac{1}{2} B_{h^{1 / 2}}\left(x_{0}\right), l_{q, r}(0)\right)>\epsilon_{0} h^{1 / 2}-C_{0} h-2 \epsilon>\frac{\epsilon_{0}}{2} h^{1 / 2}
$$

with some $\epsilon_{0}=\epsilon_{0}(\nu, \gamma)>0, h<\frac{\epsilon_{0}^{2}}{4 C_{0}^{2}}$ and $\epsilon<\frac{h^{1 / 2} \epsilon_{0}}{2}$. This contradicts (5.6) and the fact that $d\left(\Gamma\left(u^{\epsilon_{i}}\right),\left(x_{0}, t_{0}\right)\right) \rightarrow 0$ as $\epsilon_{i} \rightarrow 0$.

3. Next suppose $u_{2}-\phi$ has a strict local minimum zero in $\Omega\left(u_{2}\right)$ at $\left(x_{0}, t_{0}\right) \in \Gamma\left(u_{2}\right)$ with $|D \phi|\left(x_{0}, t_{0}\right) \neq 0$ and

$$
\max \left(\phi_{t}-\Delta \phi, \phi_{t}-r(\nu)|D \phi|^{2}\right)\left(x_{0}, t_{0}\right)<0,
$$


where $\nu=\frac{-D \phi}{|D \phi|}\left(x_{0}, t_{0}\right)$, in a local neighborhood $B_{k}\left(x_{0}\right) \times\left[t_{0}-k, t_{0}\right]$. Again for simplicity we may assume that $|D \phi|\left(x_{0}, t_{0}\right)=1$ and the maximum is strict. Due to Lemma 5.5,

$$
\Omega\left(u_{2}\right)=\liminf _{\epsilon \rightarrow 0} \Omega\left(u^{\epsilon}\right) .
$$

Therefore along a subsequence $\epsilon_{i} \rightarrow 0 u^{\epsilon_{i}}-\phi$ has its minimum in $B_{k}\left(x_{0}\right) \times$ $\left[t_{0}-k, t_{0}\right]$ at $\left(x_{i}, t_{i}\right) \in \Gamma\left(u^{\epsilon_{i}}\right)$ with $\epsilon_{i} \rightarrow 0,\left(x_{i}, t_{i}\right) \rightarrow\left(x_{0}, t_{0}\right)$ as $i \rightarrow \infty$.

Let $\alpha_{n}:=\frac{\epsilon_{n}}{\epsilon_{1}}$ and consider

$$
\begin{aligned}
& v(x, t):=\lim _{n \rightarrow \infty, r \rightarrow 0} \inf \left\{v_{n}(y, s):|(y, s)-(x, t)| \leq r\right\} \\
& v_{n}(x, t):=\alpha_{n}^{-1} u^{\epsilon_{n}}\left(\alpha_{n}\left(x-x_{\epsilon_{n}}\right), \alpha_{n}\left(t-t_{n}\right)\right)
\end{aligned}
$$

where $x_{\epsilon_{n}} \in \epsilon_{1} \mathbb{Z}^{n}$ and $\left|x_{n}-x_{\epsilon_{n}}\right| \leq \epsilon_{1}$.

We claim that $v(x, t)$ is a supersolution of $(P)_{\epsilon_{1}}$ in $B_{1}(0) \times[0,1]$.

Proof of the claim: Suppose $v-\phi$ has local (strict) minimum in $\bar{\Omega}(v))$ at $\left(x_{0}, t_{0}\right)$. Since $v \geq 0$, in fact the minimum is strict in the local neighborhood of $\left(x_{0}, t_{0}\right)$ in $\mathbb{R}^{n+1}$. (Note that this argument does not apply for the corresponding claim with subsolutions.) Without loss of generality we may assume that $|q|=1$. Hence along a subsequence $n \rightarrow \infty, v_{n}-\phi$ has a local minimum at $\left(y_{n}, s_{n}\right)$ with $\left(y_{n}, s_{n}\right) \rightarrow\left(x_{0}, t_{0}\right)$ as $n \rightarrow \infty$.

If $\left(x_{0}, t_{0}\right) \in \Omega(v)$, then $\left(y_{n}, s_{n}\right) \in \Omega\left(v_{n}\right)$ for large $n$. By definition of $v_{n}$

$$
\left(\alpha_{n} \phi_{t}-\Delta \phi\right)\left(y_{n}, s_{n}\right) \geq 0,
$$

and therefore $-\Delta \phi\left(x_{0}, t_{0}\right) \geq 0$.

If $\left(x_{0}, t_{0}\right) \in \Gamma(v)$ with $|D \phi|\left(x_{0}, t_{0}\right) \neq 0$, then either there is a sequence $\left(y_{n}, s_{n}\right) \in \Gamma\left(v_{n}\right)$ or $\left(y_{n}, s_{n}\right) \in \Omega\left(v_{n}\right)$ converging to $\left(x_{0}, t_{0}\right)$. In either case, it follows that

$$
\max \left(\alpha_{n} \phi_{t}-\Delta \phi, \phi_{t}-g\left(\frac{y_{n}}{\epsilon_{1}}\right)|D \phi|^{2}\right)\left(y_{n}, s_{n}\right) \geq 0,
$$

and thus in the limit one obtains the desired inequality at $\left(x_{0}, t_{0}\right)$ and the claim is proved.

Therefore $v$ is a supersolution of $(P)_{\epsilon_{1}}$ in $B_{1}(0) \times[-1,0]$ with

$$
v \geq P_{\nu, r}\left(x-\bar{x}, t-t_{0}\right) \text { in } B_{1}(0) \times[-1,0],
$$

where $\bar{x} \in \epsilon_{1} \mathbb{Z}^{n}$ with $\left|x_{0}-\bar{x}\right| \leq \epsilon_{1}$. This contradicts Corollary 3.9 if $\epsilon_{1}$ is sufficiently small. 


\section{References}

[A] T. Apostol, Mathematical Analysis, second edition. Addison-Wesley Publishing Company, Inc.

[BH] H. Berestycki and F. Hamel, Front propagation in periodic excitable media, Comm. Pure Appl. Math. (2002).

[C] L. A. Caffarelli, A note on Nonlinear Homogenization, Comm. pure. Appl. Math. (1999), 829-838.

[CIL] M. Crandall, H. Ishii and P. Lions, User's guide to viscosity solutions of second order differential equations, Bull. Amer. Math. Soc. 27 (1992), 1-67.

[CLM1] L. A. Caffarelli, K. Lee and A. Mellet, Singular limit and homogenization for flame propagation in periodic excitable media, Arch. Ration. Mech. Anal. 172 (2004), 153-190.

[CLM2] L. A. Caffarelli, K. Lee and A. Mellet, Homogenization and flame propagation in periodic excitable media: the asymptotic speed of propagation, Comm. Pure. Appl. Math, to appear.

[CM1] L. A. Caffarelli and A. Mellet, Capillary drops on an inhomogeneous surface, submitted, 2005.

[CM2] L. A. Caffarelli and A. Mellet, Capillary drops on an inhomogeneous surface: Contact angle hysteresis, preprint, 2005.

[CSW] L. A. Caffarelli, P. E. Souganidis and L. Wang, Homogenization of fully nonlinear, uniformly elliptic and parabolic partial differential equations in stationary ergodic media, Comm. Pure Appl. Math.58 (2005), 319-361.

[DM] G. Dal Maso and L. Modica, Nonlinear stochastic homogenization and ergodic theory, J. Reine Angew. Math. 368 (1986), 28-42.

[E] L. C. Evans, Periodic homogenisation of certain fully nonlinear partial differential equation, Proc. Roy. Soc. Edinburgh Sect. A. 120 (1992), 245-265.

[G] K. Glasner, Homogenization of contact line dynamics, submitted.

[K1] I. Kim, Uniqueness and Existence result of Hele-Shaw and Stefan problem, Arch. Rat. Mech.Anal.168 (2003), pp. 299-328. 
[K2] I. Kim, Regularity of the free boundary for the one phase Hele-Shaw problem, J. Diff. Equations, in press.

[Ko] S. M. Kozlov, The method of averaging and walk in inhomogeneous environments, Russian Math. Surveys 40(1985), 73-145.

[P] M. Primicerio, Stefan-like problems with space-dependent latent heat, Meccanica 5 (1970), 187-190.

[PV] Papanicolaou and Varadhan, Boundary value problems with rapidly oscillating random coefficients, Proceed. Colloq. on Random Fields, Rigours results in statistical mechanics and quantum field theory, J. Fritz, J.L. Lebaritz, D. Szasz (editors), Colloquia Mathematica Societ. Janos Boyai 10 (1979) 835-873.

[R] T. Roubíček, The Stefan problem in heterogeneous media, Ann. Inst. H. Poincare Anal. Non Lineaire, 6 (1989), 481-501

[VSKP] V. R. Voeller, J. B. Swenson, W. Kim and C. Paola, A Fixed grid Method for Moving Boundary Problems on the Earths Surface, European Congress on Computational Methods in Applied Sciences and Engineering, ECCOMAS 2004. 